\title{
A SUB-PRODUCT CONSTRUCTION OF POINCARÉ-EINSTEIN METRICS
}

\author{
A. ROD GOVER AND FELIPE LEITNER
}

\begin{abstract}
Given any two Einstein (pseudo-)metrics, with scalar curvatures suitably related, we give an explicit construction of a PoincaréEinstein (pseudo-)metric with conformal infinity the conformal class of the product of the initial metrics. We show that these metrics are equivalent to ambient metrics for the given conformal structure. The ambient metrics have holonomy that agrees with the conformal holonomy. In the generic case the ambient metric arises directly as a product of the metric cones over the original Einstein spaces. In general the conformal infinity of the Poincaré metrics we construct is not Einstein, and so this describes a class of non-conformally Einstein metrics for which the (Fefferman-Graham) obstruction tensor vanishes.
\end{abstract}

\section{INTRODUCTION}

Einstein metrics have a distinguished history in geometry and physics. An area of intense recent interest has been the study of conformally compact Einstein metrics and their asymptotically Einstein generalisations. In particular, there have been exciting recent developments relating the topology and scattering theory of these structures with Branson's Q-curvature, renormalised volume and related quantities [1, 2, 11, 13, 20, 25. This programme is intimately linked to the AdS/CFT programme of Physics [32, 24] which seeks to relate conformal field theory of the boundary conformal manifold to the (pseudo-)Riemannian field theory of the interior "bulk" structure.

Let $\underline{M}$ be a compact smooth manifold with boundary $M=\partial \underline{M}$. A metric $\overline{g^{+}}$on the interior $M^{+}$of $\underline{M}$ is said to be conformally compact if it extends (with some specified regularity) to $\underline{M}$ by $g=r^{2} g^{+}$where $g$ is nondegenerate up to the boundary, and $r$ is a non-negative defining function for the boundary (i.e. $M$ is the zero set for $r$, and $d r$ is non-vanishing along $M)$. In this situation the metric $g^{+}$is complete and the restriction of $g$ to $T M$ in $\left.T \underline{M}\right|_{M}$ determines a conformal structure that is independent of the choice of defining function $r$; the latter is termed the conformal infinity of $M^{+}$[26]. If the defining function is chosen so that $|d r|=1$ (with respect to $\underline{g}$ ) along $M$ then the sectional curvatures tend to -1 at infinity and the structure is said to be asymptotically hyperbolic. The model is the Poincaré hyperbolic ball. More generally one may suppose that the interior metric $g^{+}$ is Einstein in the sense that $\operatorname{Ric}\left(g^{+}\right)=-n g^{+}$, and in this case the structure is said to be Poincaré-Einstein.

A central question is the existence and uniqueness of an interior PoincaréEinstein structure $\left(M^{+}, g^{+}\right)$for a given conformal manifold $(M,[g])$. In [23] Graham-Lee showed that each conformal structure on $S^{n}$, sufficiently near 
the standard one, is the conformal infinity of a unique (up to diffeomorphism) asymptotically hyperbolic Poincaré-Einstein metric on the ball near the hyperbolic metric. This idea has been extended to more general circumstances by Biquard [7], Lee [27] and Anderson, e.g. 2]. In another direction, yielding further examples, a connected sum theory for combining PoincaréEinstein metrics has been developed by Mazzeo and Pacard [33].

The problem of obtaining existence and examples is already interesting if we drop the requirement of compactness and simply seek a Poincaré-Einstein collar (and certain extensions thereof) for a given conformal $\left(M^{n},[g]\right)$. We will use the term Poincaré-Einstein to include structures of this type, that is structures satisfying the conditions above except that we will not assume $\underline{M}$ is compact. This perspective is given without apology; in the setting of physics, for example, it is not necessarily expected that the important metrics are conformally compact. Given $\left(M^{n},[g]\right)$ with $n$ odd and $g$ analytic, a collar solution is guaranteed by the formal theory, but if $n$ is even then a formal Poincaré-Einstein metric is obstructed by a symmetric tracefree 2-tensor $\mathcal{B}[12,19,22$. In dimension 4 this is the Bach tensor from Bach's relativity [5]; in general even dimensions we will refer to this as the (Fefferman-Graham) obstruction tensor.

In this article the main result is a construction of Poincaré-Einstein metrics for a class of boundary conformal structures. More precisely it is this. Given a pair of Einstein manifolds $\left(M_{1}^{m_{1}}, g_{1}\right)$ and $\left(M_{2}^{m_{2}}, g_{2}\right)$, of signatures resp. $\left(p_{1}, q_{1}\right)$ and $\left(p_{2}, q_{2}\right)$, such that their scalar curvatures are related by $m_{2}\left(m_{2}-1\right) \operatorname{Sc}\left(g_{1}\right)=-m_{1}\left(m_{1}-1\right) \operatorname{Sc}\left(g_{2}\right)$, we give a signature $\left(p_{1}+p_{2}+1, q_{1}+q_{2}\right)$ Poincaré-Einstein structure on $M_{1} \times M_{2} \times I$, where $I$ is a suitable subset of the real line and contains an interval $\left[0, r_{0}\right)$ for some $r_{0}>0$. The conformal infinity is $\left(M_{1} \times M_{2},\left[g_{1} \times g_{2}\right]\right)$. This is Theorem 4.1. Using $r$ for the standard coordinate on the interval $\left[0, r_{0}\right)$, explicitly the interior metric is

$$
g^{+}=r^{-2}\left(d r^{2}+\left(1-\mu r^{2} / 2\right)^{2} g_{1}+\left(1+\mu r^{2} / 2\right)^{2} g_{2}\right)
$$

where $\mu$ is any constant satisfying $2 m_{1}\left(m_{1}-1\right) \mu=\operatorname{Sc}\left(g_{1}\right)$ and $2 m_{2}\left(m_{2}-\right.$ 1) $\mu:=-\operatorname{Sc}\left(g_{2}\right)$. We take $m_{1} \geq 1$ and $m_{2} \geq 0$. In the construction $m_{2}=$ 0 corresponds to taking $\left(M_{2}^{m_{2}}, g_{2}\right)$ as a point (or a collection of isolated points). Thus, as a special case, we recover an explicit Poincaré metric for the case that the boundary metric is Einstein. In the generic situation of our construction, the product metric $g_{1} \times g_{2}$ will sit uniquely as the only metric in its conformal class $\left[g_{1} \times g_{2}\right]$ which is a product of Einstein metrics, and so in this setting the Poincaré-metric is determined by the boundary conformal structure.

Given Einstein metrics $\left(M_{3}^{m_{3}}, g_{3}\right)$ and $\left(M_{4}^{m_{4}}, g_{4}\right)$, of signatures resp. $\left(p_{3}, q_{3}\right)$ and $\left(p_{4}, q_{4}\right)$ and with non-zero scalar curvature of the same sign $\varepsilon$, then

$$
g_{1}:=\varepsilon\left(m_{4} \operatorname{Sc}\left(g_{3}\right) g_{3}+m_{3} \operatorname{Sc}\left(g_{4}\right) g_{4}\right)
$$

is an Einstein metric of scalar curvature

$$
\operatorname{Sc}\left(g_{1}\right)=\varepsilon\left(\frac{m_{3}+m_{4}}{m_{3} m_{4}}\right) .
$$

Using this with the formula for $g^{+}$(with $m_{2}=0$ ), yields a Poincaré metric of signature $\left(p_{3}+p_{4}+1, q_{3}+q_{4}\right)$. Since a constant dilation $g_{1} \mapsto \alpha g_{2}$ induces 
$\operatorname{Sc}\left(g_{2}\right) \mapsto \operatorname{Sc}\left(\alpha g_{2}\right)=\frac{1}{\alpha} \operatorname{Sc}\left(g_{2}\right)$, we may summarise as follows. Given any pair of non-Ricci flat Einstein metrics $g_{1}$ and $g_{2}$, of signatures $\left(p_{1}, q_{1}\right)$ and $\left(p_{2}, q_{2}\right)$ then one obtains, via the construction, a Poincaré metric of signature $\left(p_{1}+p_{2}+1, q_{1}+q_{2}\right)$ and with conformal infinity $\left(M_{1} \times M_{2},\left[g_{1} \times \alpha g_{2}\right]\right)$, where $\alpha g_{2}$ is an appropriate constant dilation of $g_{2}$.

In section [5 we show (cf. [23]) that a certain smooth extension of the metric cone of the interior of a Poincaré-Einstein structure, with conformal infinity $(M,[g])$, yields a Ricci-flat ambient metric for $(M,[g])$ (in the sense of [12, 9] except here we obtain the ambient as a manifold with boundary as a fibered structure over the full Poincaré-Einstein structure). Thus our results above can be rephrased in terms of the ambient metric. In fact our construction proceeds in the other direction. Over a non-Ricci flat Einstein $m$-manifold $(M, g)$ one may construct the Ricci-flat dimension $m+1$ metric cone $(\bar{M}, \bar{g})$. This is a standard construction which dates back to the work of Ernst Ruh in the 1970s, see [34] for references. If $\bar{M}_{1}$ and $\bar{M}_{2}$ are two such cones, over Einstein metrics $g_{1}$ and $g_{2}$ with an appropriate scalar curvature relation, then we show, in section 3 that the product $\left(\bar{M}_{1} \times \bar{M}_{2}, \bar{g}_{1} \times \overline{g_{2}}\right)$ is an ambient metric for $\left(M_{1} \times M_{2},\left[g_{1} \times g_{2}\right]\right)$. The construction generalises in the sense that one may write down the same ambient metric directly and this then extends to the case that $g_{1}$ and $g_{2}$ are Ricci-flat. This general case is treated first in Theorem 2.1, of section 2, where we verify explicitly that the metric satisfies the conditions of a Ricci-flat ambient metric as in [9, 12. The Poincaré-Einstein metric arises as the induced structure on a certain codimension 1 submanifold of the ambient manifold, via a construction of Fefferman-Graham [12] (see also [14]). Thus Poincaré-Einstein metrics are equivalent to ambient metrics, at least ambient metrics as manifolds with boundary, in the sense of section 5 .

Recently there has been considerable interest in conformal holonomy 3 , 29, 31, 30, 28, that is the holonomy of the normal conformal tractor (or Cartan) connection of [10, 6, 8]. In Theorem 6.4 we show that holonomy of the ambient metrics from Theorem 2.1 agrees with the conformal holonomy. Using this result we show in Theorem 3.4 that in general the products $g_{1} \times$ $g_{2}$ where $m_{2}\left(m_{2}-1\right) \operatorname{Sc}\left(g_{1}\right)=-m_{1}\left(m_{1}-1\right) \operatorname{Sc}\left(g_{2}\right)$, are not conformally Einstein. In fact we show the stronger result that they are in general not conformally almost-Einstein in the sense of [16]. This shows that the general construction of ambient metrics and Poincaré-Einstein metrics here is not a disguised form of the simpler construction for Einstein boundaries. The ambient variant of the latter seems to have been first given in [30, 22] (and see also [3]). Since the obstruction tensor $\mathcal{B}$ is an obstruction to the type of ambient metrics and Poincaré metrics that we construct [19, 22], it also follows that the generic products of this form give a large class of metrics which are not conformally almost-Einstein and yet for which the obstruction tensor $\mathcal{B}$, mentioned above, vanishes, see Corollary 3.3 and Theorem 3.4 . (The obstruction tensor vanishes on manifolds which are conformally almostEinstein [12, 19, 22].)

In section 7 we show that the Poincaré-Einstein interior metrics, that we obtain, are characterised by the presence of so-called special Killing 
forms. These are simple Killing forms satisfying additional integrability conditions as described in Theorem 17.2. In the final section we give examples of Poincaré-Einstein metrics where the boundary conformal structure is not conformally Einstein. It is also observed there that one can obviously iterate the construction of Poincaré-Einstein metrics, as described in Theorem 4.1 , to obtain a recursive construction principle for a class of Poincaré-Einstein metrics.

We have noticed that Armstrong and Leistner have just developed 4 an interesting construction with some relation to that we give in section 2 , They give a construction of an ambient type space with a connection with torsion the Cotton tensor.

ARG gratefully acknowledges support from the Royal Society of New Zealand via Marsden Grant no. 02-UOA-108, and to the New Zealand Institute of Mathematics and its Applications for 2004 support via a Maclaurin Fellowship. FL would like to thank the University of Auckland for support in 2004 when the initial construction was developed. ARG and FL would also like to express thanks to: Hans-Bert Rademacher and the Graduiertenkolleg "Analysis, Geometry and its Interaction with the Natural Sciences" at Universität Leipzig; Wolfgang Kühnel and the University of Stuttgart; Helga Baum, and the Humboldt-Universität Berlin; the Institut des Hautes Étudies Scientifiques; and the Institute for Mathematics and its Applications, Minnesota.

\section{Ambient metrics}

Let $M$ be a smooth $n$-manifold. Recall that a conformal structure of signature $(p, q)$ on $M$ is a smooth ray subbundle $S^{2} T^{*} M \supset \mathcal{Q} \stackrel{\pi}{\rightarrow} M$ whose fibre over $x$ consists of conformally related signature- $(p, q)$ metrics at the point $x$. Sections of $\mathcal{Q}$ are metrics $g$ on $M$. So we may equivalently view the conformal structure as the equivalence class $[g]$ of these conformally related metrics. Let us use $\rho$ to denote the $\mathbb{R}_{+}$action on $\mathcal{Q}$ given by $\rho(s)\left(x, g_{x}\right)=$ $\left(x, s^{2} g_{x}\right)$. Following [9], an ambient manifold is to mean a smooth $(n+$ 2)-manifold $\tilde{M}$ endowed with a free $\mathbb{R}_{+}$-action $\rho$ and an $\mathbb{R}_{+}$-equivariant embedding $i: \mathcal{Q} \rightarrow \tilde{M}$. We write $\boldsymbol{X} \in \mathfrak{X}(\tilde{M})$ for the fundamental field generating the $\mathbb{R}_{+}$-action, that is for $f \in C^{\infty}(\tilde{M})$ and $u \in \tilde{M}$ we have $\boldsymbol{X} f(u)=\left.(d / d t) f\left(\rho\left(e^{t}\right) u\right)\right|_{t=0}$.

If $i: \mathcal{Q} \rightarrow \tilde{M}$ is an ambient manifold, then an ambient metric is a pseudoRiemannian metric $\boldsymbol{h}$ of signature $(p+1, q+1)$ on $\tilde{M}$ such that the following conditions hold:

(i) The metric $\boldsymbol{h}$ is homogeneous of degree 2 with respect to the $\mathbb{R}_{+}$-action, i.e. if $\mathcal{L}_{\boldsymbol{X}}$ denotes the Lie derivative by $\boldsymbol{X}$, then we have $\mathcal{L}_{\boldsymbol{X}} \boldsymbol{h}=2 \boldsymbol{h}$. (I.e. $\boldsymbol{X}$ is a homothetic vector field for $h$.)

(ii) For $u=\left(x, g_{x}\right) \in \mathcal{Q}$ and $\xi, \eta \in T_{u} \mathcal{Q}$, we have $\boldsymbol{h}\left(i_{*} \xi, i_{*} \eta\right)=g_{x}\left(\pi_{*} \xi, \pi_{*} \eta\right)$.

To simplify the notation we will usually identify $\mathcal{Q}$ with its image in $\tilde{M}$ and suppress the embedding map $i$. To link the geometry of the ambient manifold to the underlying conformal structure on $M$ one requires further conditions. In [12] Fefferman and Graham treat the problem of constructing 
a formal power series solution along $\mathcal{Q}$ for the (Goursat) problem of finding an ambient metric $\boldsymbol{h}$ satisfying (i) and (ii) and the condition that it be Ricci flat, i.e. $\operatorname{Ric}(\boldsymbol{h})=0$. A key result is Theorem 2.1 of their paper: If $n$ is odd, then up to a $\mathbb{R}_{+}$-equivariant diffeomorphism fixing $\mathcal{Q}$, there is a unique power series solution for $\boldsymbol{h}$ satisfying (i), (ii) and $\operatorname{Ric}(\boldsymbol{h})=0$. If $n$ is even, then in general one may formally obtain $\operatorname{Ric}(\boldsymbol{h})=0$ only up to the addition of terms vanishing to order $n / 2-1$. See [18, 19, 21] for further discussion.

For a pair of suitable Einstein metrics $g_{1}$ and $g_{2}$ we give here an explicit Ricci-flat ambient metric. In the theorem we include the case of just a single Einstein manifold $M_{1}$. This is consistent with the general construction by taking the view that the second manifold $M_{2}$ is a single point (and so of dimension $m_{2}=0$ ). It was known to Fefferman and Graham [12] that the problem of constructing a formal ambient metric was solvable to all orders whenever the conformal structure underlying manifold $M$ was conformally Einstein. In 30 an explicit ambient metric was given for that case. The following theorem may be viewed as an extension and generalisation of those results.

Theorem 2.1. Suppose that $\left(M_{1}^{m_{1}}, g_{1}\right)$ and $\left(M_{2}^{m_{2}}, g_{2}\right)$, of signatures resp. $\left(p_{1}, q_{1}\right)$ and $\left(p_{2}, q_{2}\right)$, and with $m_{1} \geq 1, m_{2} \geq 0$, are Einstein manifolds such that $m_{2}\left(m_{2}-1\right) \operatorname{Sc}\left(g_{1}\right)=-m_{1}\left(m_{1}-1\right) \operatorname{Sc}\left(g_{2}\right)$. For each $\mu \in \mathbb{R}$ satisfying $2 m_{1}\left(m_{1}-1\right) \mu=\operatorname{Sc}\left(g_{1}\right)$ and $2 m_{2}\left(m_{2}-1\right) \mu=-\operatorname{Sc}\left(g_{2}\right)$, there is a signature $\left(p_{1}+p_{2}+1, q_{1}+q_{2}+1\right)$ Ricci-flat ambient manifold for the conformal manifold $\left(M_{1} \times M_{2},\left[g_{1} \times g_{2}\right]\right)$, with metric given by the expression (11) below.

Note that if either of $m_{1}$ or $m_{2}$ is at least 2 then there is exactly one solution $\mu$ to the condition, $2 m_{1}\left(m_{1}-1\right) \mu=\operatorname{Sc}\left(g_{1}\right)$ and $2 m_{2}\left(m_{2}-1\right) \mu=-\operatorname{Sc}\left(g_{2}\right)$. Otherwise $\mu$ is any real number. (In fact if $m_{1}=1$ and $m_{2}=0$ then $\mu$ can be taken to be a non-vanishing function. We do not treat this as a special case as the factor may absorbed as a conformal transformation of $g_{1}$.)

Proof of the Theorem: Let us simplify notation by defining $M:=M_{1} \times$ $M_{2}$, and $g:=g_{1} \times g_{2}$. We write $\pi: \mathcal{Q} \rightarrow M$ for the $\mathbb{R}_{+}$-bundle of metrics conformally related to $g$. The metric $g$ determines a fibre coordinate $t$ on $\mathcal{Q}$ by writing a general point of $\mathcal{Q}$ in the form $\left(p, t^{2} g(p)\right)$, where $p \in M$ and $t>0$.

The ambient manifold is defined to be $\tilde{M}:=\mathcal{Q} \times \tilde{I}$ where

$$
\tilde{I}= \begin{cases}\mathbb{R} & \text { if } \mu=0, \\ \mathbb{R} \backslash\left\{-\frac{1}{\mu}\right\} & \text { if } \mu \neq 0 \text { and } m_{2}=0, \\ \mathbb{R} \backslash\left\{\frac{1}{\mu},-\frac{1}{\mu}\right\} & \text { otherwise. }\end{cases}
$$

There is a projection $\tilde{M} \rightarrow \mathcal{Q}$ given by forgetting the $\tilde{I}$ component in the product. Following this with $\pi: \mathcal{Q} \rightarrow M$ we have a projection $\tilde{\pi}: \tilde{M} \rightarrow M$. It follows that we have the canonical bilinear forms $\tilde{\pi}^{*} g_{1}$ and $\tilde{\pi}^{*} g_{2}$ on $\tilde{M}$. For notational simplicity let us write, respectively, $g_{1}$ and $g_{2}$ for these forms on $\tilde{M}$.

We equip $\tilde{M}$ with the metric

$$
\boldsymbol{h}:=2 t d t d \rho+2 \rho d t^{2}+t^{2}\left[(1+\mu \rho)^{2} g_{1}+(1-\mu \rho)^{2} g_{2}\right],
$$


where $\rho$ is the standard coordinate on $\mathbb{R}$ viewed as a coordinate on $\tilde{I}$ (and hence on $\tilde{M}$ ) and $\mathcal{Q}$ is identified with its image $\mathcal{Q} \times\{0\} \subset \tilde{M}$. Note that $\rho$ is a defining function for $\mathcal{Q}$ and that the functions $1 \pm \mu \rho$ are non-vanishing on the set $\tilde{I}$. Observe that the $\mathbb{R}_{+}$action on $\mathcal{Q}$ extends to $\tilde{M}$ in the obvious way via the product $\mathcal{Q} \times \tilde{I}$. Thus $\boldsymbol{X}:=t \partial_{t}$ extends the fundamental vector field on $\mathcal{Q}$ and it is clear that $\mathcal{L}_{\boldsymbol{X}} \boldsymbol{h}=2 \boldsymbol{h}$. Since $\rho$ is a defining function for $\mathcal{Q}$ it is clear that along $\mathcal{Q}$, and upon restriction to $T \mathcal{Q}, \boldsymbol{h}$ agrees with the tautological bilinear form on $\mathcal{Q}$ determined by $[g]$. That is we have the property (ii) for an ambient metric.

It remains to check that $\boldsymbol{h}$ is Ricci flat. Fixing some choice of local coordinates $x^{1}, \cdots, x^{m_{1}}$ on $M_{1}$ and $x^{m_{1}+1}, \cdots, x^{m_{1}+m_{2}}$ on $M_{2}$, the coordinates $(x, t, \rho)$ on $\tilde{M}$ are the obvious extension of the coordinates $(x, t)$ on $\mathcal{Q}$. We calculate in these coordinates, and for a function $f(x, t, \rho)$ the notation $f^{\prime}$ will mean $\partial f / \partial \rho$. For any metric $\boldsymbol{h}$ in the form (cf. [12])

$$
\boldsymbol{h}=2 t d t d \rho+2 \rho d t^{2}+t^{2} \tilde{g}_{i j}(x, \rho) d x^{i} d x^{j},
$$

where $\tilde{g}_{i j}(x, \rho)$ is (the pull-back to $\tilde{M}$ of) a 1-parameter family of metrics on $M$, we have the following: $\boldsymbol{X}$ is homothetic and $\boldsymbol{h}(\boldsymbol{X}, \cdot)$ is closed (in fact exact) and so $\boldsymbol{X}^{A} \operatorname{Ric}(\boldsymbol{h})_{A B}=0$ (whence $\left.\operatorname{Ric}(\boldsymbol{h})_{t i}=0=\operatorname{Ric}(\boldsymbol{h})_{t t}\right) ; \operatorname{Ric}(\boldsymbol{h})_{i j}$ is (the pull-back to $\tilde{M}$ of) the tensor

$$
\rho \tilde{g}_{i j}^{\prime \prime}-\rho \tilde{g}^{k l} \tilde{g}_{i k}^{\prime} \tilde{g}_{j l}^{\prime}+\frac{1}{2} \rho \tilde{g}^{k l} \tilde{g}_{k l}^{\prime} \tilde{g}_{i j}^{\prime}+\frac{2-n}{2} \tilde{g}_{i j}^{\prime}-\frac{1}{2} \tilde{g}^{k l} \tilde{g}_{k l}^{\prime} \tilde{g}_{i j}+\operatorname{Ric}(\tilde{g})_{i j},
$$

on $M ; \operatorname{Ric}(\boldsymbol{h})_{\rho \rho}$ is

$$
-\frac{1}{2} \tilde{g}^{i j} \tilde{g}_{i j}^{\prime \prime}+\frac{1}{4} \tilde{g}^{i j} \tilde{g}^{k l} \tilde{g}_{i k}^{\prime} \tilde{g}_{j l}^{\prime}
$$

and $\operatorname{Ric}(\boldsymbol{h})_{\rho j}$ is

$$
\tilde{\nabla}_{\ell}^{(\rho)}\left(\tilde{g}^{k \ell} \tilde{g}_{k j}^{\prime}\right)-\tilde{\nabla}_{j}^{(\rho)}\left(\tilde{g}^{k \ell} \tilde{g}_{k \ell}^{\prime}\right),
$$

where, for each value of the parameter $\rho, \tilde{\nabla}^{(\rho)}$ is the Levi-Civita covariant derivative on $M$ for $\tilde{g}(x, \rho)$. Now we calculate each of these in turn.

First we calculate (3), (4), and (5) for the metric (11) in the case that $m_{2}=0$, that is $\tilde{g}=a^{2} g_{1}$ where $a:=1+\mu \rho$. For simplicity write $n=m_{1}$ and $g=g_{1}$. Then we have $\tilde{g}_{i j}^{\prime}=2 \mu a g_{i j}$ and hence we have $\tilde{g}^{k l} \tilde{g}_{k l}^{\prime}=2 \mu a^{-1} n$ and $\tilde{g}_{i j}^{\prime \prime}=2 \mu^{2} g_{i j}$. Substituting these in (3) we obtain

$$
\begin{aligned}
& -2 \rho \mu^{2}+2 n \rho \mu^{2}+(2-n) a \mu-a n \mu+2 n \mu-2 \mu \\
& =\mu(n-1)(2 \rho \mu-2 a+2)=0
\end{aligned}
$$

since $\rho \mu=a-1$. For (4) we need also $\tilde{g}^{i j} \tilde{g}_{i j}^{\prime \prime}=2 a^{-2} \mu^{2} n$ and substituting this gives $-n a^{-2} \mu^{2}+n a^{-2} \mu^{2}=0$. Finally for (5) observe that $\tilde{g}^{k \ell} \tilde{g}_{k \ell}^{\prime}$ depends only on $\rho$ and so $\tilde{\nabla}_{j}^{(\rho)}\left(\tilde{g}^{k \ell} \tilde{g}_{k \ell}^{\prime}\right)=0$. On the other hand $\tilde{g}^{k \ell} \tilde{g}_{k j}^{\prime}=2 \mu a^{-1} \delta_{j}^{\ell}$, Thus

$$
\tilde{\nabla}_{\ell}^{(\rho)}\left(\tilde{g}^{k \ell} \tilde{g}_{k j}^{\prime}\right)=2 \mu a^{-1} \tilde{\nabla}_{\ell}^{(\rho)} \delta_{j}^{\ell}=0
$$

and so $\operatorname{Ric}(\boldsymbol{h})_{\rho j}=0$.

Next we assume $m_{2} \geq 1$ and so $\tilde{g}=a^{2} g_{1}+b^{2} g_{2}$, where $a:=(1+\mu \rho)$ and $b:=(1-\mu \rho)$. First note that $\tilde{g}_{i j}^{\prime}=2 \mu a g_{i j}^{1}-2 \mu b g_{i j}^{2}$ and hence we have 
$\tilde{g}^{k l} \tilde{g}_{k l}^{\prime}=2 \mu\left(a^{-1} m_{1}-b^{-1} m_{2}\right)$ and $\tilde{g}_{i j}^{\prime \prime}=2 \mu^{2} g_{i j}^{1}+2 \mu^{2} g_{i j}^{2}$. Substituting these in (3) and assuming that $1 \leq i \leq m_{1}$ brings us to

$-2 \rho \mu^{2}+2 \rho \mu^{2}\left(m_{1}-m_{2} \frac{a}{b}\right)+\left(2-m_{1}-m_{2}\right) \mu a-\mu a\left(m_{1}-m_{2} \frac{a}{b}\right)+\frac{1}{m_{1}} \operatorname{Sc}\left(g_{1}\right)$

times $g_{i j}^{1}$. But now using that $\operatorname{Sc}\left(g_{1}\right)=2 m_{1}\left(m_{1}-1\right) \mu$ and $a-b=2 \mu \rho$ this becomes

$$
\begin{aligned}
& \frac{\mu}{b}\left[b(b-a)+(a-b)\left(b m_{1}-a m_{2}\right)\right. \\
& \left.+a b\left(2-m_{1}-m_{2}\right)-a\left(b m_{1}-a m_{2}\right)+2 b\left(m_{1}-1\right)\right]=\mu(b+a-2)\left(1-m_{1}\right)
\end{aligned}
$$

which vanishes identically since $b+a=2$. A similar calculation for (3) with $m_{1}+1 \leq i \leq m_{1}+m_{2}$ gives $\mu(b+a-2)\left(1-m_{2}\right)=0$ and so $\operatorname{Ric}(\boldsymbol{h})$ vanishes identically on $\tilde{M}$.

For (4) with $\tilde{g}=a^{2} g_{1}+b^{2} g_{2}$ we have

$$
-\frac{1}{2}\left(2 \mu^{2} a^{-2} m_{1}+2 \mu^{2} b^{-2} m_{2}\right)+\mu^{2} a^{-2} m_{1}+\mu^{2} b^{-2} m_{2}=0 .
$$

Finally the case (15). First observe that $\tilde{g}^{k \ell} \tilde{g}_{k \ell}^{\prime}=2 \mu\left(a^{-1} m_{1}-b^{-1} m_{2}\right)$ depends only on $\rho$ and so $\tilde{\nabla}_{j}^{(\rho)}\left(\tilde{g}^{k \ell} \tilde{g}_{k \ell}^{\prime}\right)=0$. On the other hand $\tilde{g}^{k \ell} \tilde{g}_{k j}^{\prime}=$ $2 \mu a^{-1} P_{(1)}^{\ell}-2 \mu b^{-1} P_{(2)}^{\ell}{ }_{j}^{\ell}$, where $P_{(1)}$ is the section of $\operatorname{End}(T M)$ projecting onto $T M_{1}$ and $P_{(2)}$ is the complementary projection onto $T M_{2}$. (Here we view $T M$ as $T M_{1} \oplus T M_{2}$ via the derivative of the product structure $M=$ $M_{1} \times M_{2}$.) Thus

$$
\tilde{\nabla}_{\ell}^{(\rho)}\left(\tilde{g}^{k \ell} \tilde{g}_{k j}^{\prime}\right)=2 \mu a^{-1} \tilde{\nabla}_{\ell}^{(\rho)} P_{(1)}^{\ell}-2 \mu b^{-1} \tilde{\nabla}_{\ell}^{(\rho)} P_{(2)}^{\ell}{ }_{j}^{\ell} .
$$

But $\tilde{\nabla}^{(\rho)}$ is the Levi-Civita connection for a product metric compatible with the structure $M_{1} \times M_{2}$. Thus $\tilde{\nabla}^{(\rho)} P_{(1)}=0=\tilde{\nabla}^{(\rho)} P_{(2)}$ and we conclude that $\operatorname{Ric}(\boldsymbol{h})_{\rho j}=0$.

\section{The GeneriC SETting AND Metric CONES}

For $\left(M^{m}, g\right)$ an Einstein manifold of scalar curvature $\mathrm{Sc}(\mathrm{g}) \neq 0$ the metric cone is usually defined to be $\bar{M}=M \times \mathbb{R}_{+}$equipped with the metric

$$
s^{2} g+\frac{m(m-1)}{\mathrm{Sc}(\mathrm{g})} d s^{2} .
$$

This is Ricci-flat [34. As a minor variation on this theme we equip $\bar{M}$ with the metric

$$
\bar{g}=\operatorname{sgn}\left(\lambda^{g}\right)\left(\lambda^{g} s^{2} g+d s^{2}\right)
$$

where $\lambda^{g}$ satisfies $\operatorname{Sc}(g)=m(m-1) \lambda^{g}$. Then the metric $\bar{g}$ is well defined and Ricci-flat for all dimensions $m \geq 1$ of the base manifold. We call $(\bar{M}, \bar{g})$ the metric cone for $(M, g)$. Note that if $g$ has signature $(p, q)$ then the cone has signature $(p+1, q)$ or $(p, q+1)$ according to whether $\lambda^{g}$ is respectively positive or negative.

Now suppose that we have a pair of Einstein manifolds $\left(M_{1}^{m_{1}}, g_{1}\right)$ and $\left(M_{2}^{m_{2}}, g_{2}\right)$ such that $m_{2}\left(m_{2}-1\right) \operatorname{Sc}\left(g_{1}\right)=-m_{1}\left(m_{1}-1\right) \operatorname{Sc}\left(g_{2}\right)$ as in Theorem 2.1 (and we allow the case $m_{2}=0$ as explained there.) We will show that 
the product of the cones over $\left(M_{1} \times M_{2}, g_{1} \times g_{2}\right)$ is the ambient manifold from Theorem 2.1.

With $\lambda$ satisfying $\operatorname{Sc}\left(g_{1}\right)=m_{1}\left(m_{1}-1\right) \lambda$ there is no essential loss of generality in assuming that $\lambda>0$. Then the cone metrics are

$$
\bar{g}_{1}=\lambda s_{1}^{2} g_{1}+d s_{1}^{2} \quad \text { and } \quad \bar{g}_{2}=\lambda s_{2}^{2} g_{2}-d s_{2}^{2}
$$

on, respectively, $\bar{M}_{1}$ and $\bar{M}_{2}$. A product of Ricci-flat metrics is always Ricci-flat and so in particular this is true for the product metric

$$
\boldsymbol{h}_{\times}:=\bar{g}_{1}+\bar{g}_{2}=d s_{1}^{2}-d s_{2}^{2}+\lambda s_{1}^{2} g_{1}+\lambda s_{2}^{2} g_{2}
$$

on $\bar{M}_{1} \times \bar{M}_{2}$. Now we define functions $t$ and $\rho$ on $\bar{M}_{1} \times \bar{M}_{2}$ by

$$
t:=\frac{\lambda^{1 / 2}\left(s_{1}+s_{2}\right)}{2} \quad \text { and } \quad \rho:=\frac{2\left(s_{1}-s_{2}\right)}{\lambda\left(s_{1}+s_{2}\right)},
$$

and set $\mu=\lambda / 2$. Re-expressing the right-hand-side of (8) in terms of $t$, $\rho, \mu$, and the pull-back metrics $g_{1}$ and $g_{2}$, a direct calculation recovers exactly the expression for the ambient metric as given on the right-handside of (1). The Jacobian $\partial(t, \rho) / \partial\left(s_{1}, s_{2}\right)$ is non-vanishing on the positive $\left(s_{1}, s_{2}\right)$-quadrant and so, with the pull-back (under the obvious projections) of coordinate sets from $M_{1}$ and $M_{2}$, the pair $t, \rho$ give coordinates on the entire product $\bar{M}_{1} \times \bar{M}_{2}$. The metric (1) extends this, and since the inverse of the transformation (9) is

$$
s_{1}=(2 \mu)^{-1 / 2} t(1+\mu \rho) \text { and } s_{2}=(2 \mu)^{-1 / 2} t(1-\mu \rho),
$$

we see immediately that the points where the ambient metric (11) degenerates (e.g. $\rho= \pm \frac{1}{\mu}$ in the generic case) are points bounding but not in the product $\bar{M}_{1} \times \bar{M}_{2}$. (In stating things this way we are viewing both $\tilde{M}$ and the product $\bar{M}_{1} \times \bar{M}_{2}$ as subspaces, in the obvious way, of the manifold $\mathcal{Q} \times \mathbb{R}$.) In summary we have the following result.

Proposition 3.1. Suppose that $\left(M_{1}^{m_{1}}, g_{1}\right)$ and $\left(M_{2}^{m_{2}}, g_{2}\right)$ are Einstein manifolds such that $m_{2}\left(m_{2}-1\right) \operatorname{Sc}\left(g_{1}\right)=-m_{1}\left(m_{1}-1\right) \operatorname{Sc}\left(g_{2}\right)$. In a neighbourhood of $\mathcal{Q} \subset \tilde{M}$, the ambient metric (1) for $\left(M_{1} \times M_{2},\left[g_{1} \times g_{2}\right]\right)$ is the product of the cone metrics (7) where $\lambda=2 \mu$ satisfies $\operatorname{Sc}\left(g_{1}\right)=m_{1}\left(m_{1}-1\right) \lambda$ and $\operatorname{Sc}\left(g_{2}\right)=-m_{2}\left(m_{2}-1\right) \lambda$.

We make some observations in relation to this picture.

Proposition 3.2. The ambient metric $\boldsymbol{h}$ given in (1) is independent of constant dilations of the product metric $g_{1} \times g_{2}$ on $M_{1}^{m_{1}} \times M_{2}^{m_{2}}$.

Proof: First observe that if $\alpha \in \mathbb{R}_{+}$and metrics $g$ and $\widehat{g}$ are related by a constant conformal rescaling according to $\widehat{g}=\alpha g$ then $\operatorname{Ric}(\widehat{g})=\operatorname{Ric}(g)$. Thus $\operatorname{Sc}(\widehat{g})=\alpha^{-1} \operatorname{Sc}(g)$ and so, making the compatible transformation of $\lambda^{g}$ to $\lambda^{\widehat{g}}$, we have $\lambda^{g} g=\lambda^{\widehat{g}} \widehat{g}$ and the cone metric (6) for $(M, g)$ is the same as the cone metric for $(M, \widehat{g})$. It follows easily that the product metric $\boldsymbol{h}_{\times}$on $\bar{M}_{1} \times \bar{M}_{2}$ depends only on $g_{1} \times g_{2}$ up to dilations. But this extends to $\boldsymbol{h}$ on $\tilde{M}$ since, via (10), there is a formulae for $\boldsymbol{h}$ of the form (8) on a dense subspace of $\tilde{M}$.

Remark: From the Proposition it follows that, from the conformal point 
of view, when $\lambda \neq 0$ with $\max \left(m_{1}, m_{2}\right) \geq 2$ there is no loss of generality in setting $\mu=1$.

As a special case of Proposition 3.1 note that for an Einstein manifold $\left(M^{m}, g\right)$ of scalar curvature $m(m-1) \lambda, \lambda>0$, an ambient metric is given by

$$
\lambda s_{1}^{2} g+d s_{1}^{2}-d s_{2}^{2}
$$

on $M \times \mathbb{R}_{+} \times \mathbb{R}_{+}$. We may view this as the product of the metric cone with the cone over a point. (In fact we could allow $s_{2}$ to range over $\mathbb{R}$ but this extension is not critical for our current discussions.) There is an obvious variant of this for the case $\lambda<0$.

The observations above lead to Theorem 3.4 below. First we note that there is an obvious consequence of the ambient construction in Theorem 2.1. An ambient manifold, as described at the start of section 2, can only be Ricci-flat if the Fefferman-Graham obstruction tensor $\mathcal{B}$ is identically zero [12, 19. Thus we have the following.

Corollary 3.3. Suppose that $g$ is a metric conformally related to a product metric $g_{1} \times g_{2}$, where $\left(M_{1}^{m_{1}}, g_{1}\right)$ and $\left(M_{2}^{m_{2}}, g_{2}\right)$ are Einstein structures such that $m_{2}\left(m_{2}-1\right) \operatorname{Sc}\left(g_{1}\right)=-m_{1}\left(m_{1}-1\right) \operatorname{Sc}\left(g_{2}\right) \neq 0$. Then the obstruction tensor $\mathcal{B}^{g}$ is everywhere vanishing.

As mentioned earlier, it was already known that the obstruction tensor necessarily vanishes on manifolds that are conformally Einstein (or more generally it vanishes on conformally almost-Einstein manifolds as below). Thus part of the importance of the Corollary above is that, according to the next Theorem, it gives a more general class of structures for which the obstruction vanishes identically.

Recall that an almost-Einstein structure [16] on a manifold is a conformal structure with a parallel standard tractor. Almost Einstein structures generalise the notion of Einstein manifolds since an almost-Einstein manifold is Einstein on an open dense subspace. The parallel tractor determines the Einstein scale. The corresponding conformal notion (i.e. the corresponding generalisation of conformally Einstein) is a conformal structure that is known to admit (at least) one parallel standard tractor (but this is not specified). In this case we say the manifold is conformally almost Einstein.

Theorem 3.4. Suppose that $\left(M_{1}^{m_{1}}, g_{1}\right)$ and $\left(M_{2}^{m_{2}}, g_{2}\right)$, are Einstein structures such that $m_{2}\left(m_{2}-1\right) \operatorname{Sc}\left(g_{1}\right)=-m_{1}\left(m_{1}-1\right) \operatorname{Sc}\left(g_{2}\right) \neq 0$. Then the product metric $g_{1} \times g_{2}$ on $M_{1} \times M_{2}$ is conformally almost-Einstein if and only if either $\left[g_{1}\right]$ admits two linearly independent almost-Einstein structures or $\left[g_{2}\right]$ admits two linearly independent almost-Einstein structures.

Proof: Suppose that $\left[g_{1} \times g_{2}\right]$ is conformally almost-Einstein. Then the standard tractor bundle admits a parallel tractor $I^{A}$. From Theorem 6.4 the holonomy of the standard tractor bundle is canonically the same as the holonomy group for the ( $\mathcal{Q}$ connected component of the) ambient metric (1). Thus there is a corresponding parallel vector field $\boldsymbol{I}$ on the ambient space. Since we have a product connection on the ambient space, the projections of $\boldsymbol{I}, \operatorname{pr}_{1}(\boldsymbol{I}) \in \Gamma\left(T \bar{M}_{1}\right)$ and $p r_{2}(\boldsymbol{I}) \in \Gamma\left(T \bar{M}_{2}\right)$ are each parallel. It follows that one of these, without loss of generality $\boldsymbol{I}_{1}:=\operatorname{pr}_{1}(\boldsymbol{I})$ is not-zero. So $\bar{M}_{1}$ 
has the parallel vector field $\boldsymbol{I}_{1}$. It follows that this is clearly also parallel for the ambient metric

$$
\boldsymbol{h}_{1}:=\lambda s_{1}^{2} g_{1}+d s_{1}^{2}-d s_{2}^{2}
$$

of $M_{1}$ (c.f. (11)). Note that from the construction of $\boldsymbol{I}_{1}$ on this ambient space as a trivial extension of a vector field on the cone $\bar{M}_{1}$, it follows immediately that $d s_{2}\left(\boldsymbol{I}_{1}\right)=0$. On the other hand the vector field $V=$ $\partial / \partial s_{2}$ is also clearly parallel for $\boldsymbol{h}_{1}$ and linearly independent of $\boldsymbol{I}_{1}$ (since $\left.d s_{2}(V)=1 \neq 0\right)$. Linearly independent parallel tractors on the ambient manifold determine linearly independent parallel standard tractors for the normal tractor connection [9, 18] and so $\left(M_{1},\left[g_{1}\right]\right)$ has two almost-Einstein structures.

In the other direction. If $\left(M_{1},\left[g_{1}\right]\right)$ has two linearly independent parallel tractors then, once again using Theorem 6.4 there are two corresponding, linear independent, parallel vector fields on the ambient space $\tilde{M}_{1}$. At least one of these projects to a non-zero parallel vector field on $\bar{M}_{1}$. Then obviously this parallel field on $\bar{M}_{1}$ also yields a parallel vector field for the product metric on $\tilde{M}=\bar{M}_{1} \times \bar{M}_{2}$. Thus it determines a parallel tractor on $\left(M_{1} \times M_{2},\left[g_{1} \times g_{2}\right]\right)$.

Remark: There exist manifolds that admit exactly one Einstein structure (up to constant dilation of the metric), see section 8 for examples.

Note that it follows from the Theorem that if, for example, $\left[g_{1} \times g_{2}\right]$ admits an Einstein scale, then on one of the components, say $M_{1}$ without loss of generality, on an open dense set the conformal structure $\left[g_{1}\right]$ admits two independent Einstein scales. (Of course we may take one of these to be the Einstein scale on all of $M_{1}$ assumed in the Theorem.) Conversely if we have that $M_{1}$ admits two Einstein scales then on an open dense subset of $M_{1} \times M_{2}$ the metric $g_{1} \times g_{2}$ is conformally Einstein.

As a slight digression we point out that when there are multiple (almost-) Einstein scales then these are never isolated. Since almost-Einstein structures are exactly parallel sections of the standard normal conformal tractor bundle [16, it follows that if there are two distinct almost-Einstein structures then there is a 2-dimensional family $\left(\mathbb{R}^{2} \backslash\{0\}\right)$ of such structures.

For our later considerations we observe some basic results concerning the Euler vector field for cone products. Given a tensor field on a manifold we use the same notation for the trivial extension of this field to a field on a product of the manifold with another. For a pair of pseudo-Riemannian manifolds $\left(\bar{M}_{1}, \bar{g}_{1}\right)$ and $\left(\bar{M}_{2}, \bar{g}_{2}\right)$, the product metric $\bar{g}_{1} \times \bar{g}_{2}$ is given, using this convention, as a covariant 2-tensor on $\bar{M}_{1} \times \bar{M}_{2}$, by $\bar{g}_{1}+\bar{g}_{2}$. For a constant $\alpha$ we say a vector field $V$ is an $\alpha$-homothety of a metric $g$ if $\mathcal{L}_{V} g=\alpha g$.

Lemma 3.5. Given pseudo-Riemannian manifolds $\left(\bar{M}_{1}, \bar{g}_{1}\right)$ and $\left(\bar{M}_{2}, \bar{g}_{2}\right)$, $\boldsymbol{X}_{1}$ is an $\alpha$-homothety of $\bar{g}_{1}$ and $\boldsymbol{X}_{2}$ is an $\alpha$-homothety of $\bar{g}_{2}$ if and only if $\boldsymbol{X}_{1}+\boldsymbol{X}_{2}$ is an $\alpha$-homothety of $\left(\bar{M}_{1} \times \bar{M}_{2}, \bar{g}_{1} \times \bar{g}_{2}\right)$.

Proof: From the definition of the Lie derivative it follows immediately that, for any tensor $T$ on $\bar{M}_{1}$, its trivial extension to $\bar{M}_{1} \times \bar{M}_{2}$ satisfies the condition that $\mathcal{L}_{V} T=0$ for any vector field $V$ on $\bar{M}_{2}$. Of course we can 
swap the roles of $\bar{M}_{1}$ and $\bar{M}_{2}$ in this statement. Using this the result follows from the bilinearity and naturality of the Lie derivative:

$$
\begin{aligned}
\mathcal{L}_{\boldsymbol{X}_{1}+\boldsymbol{X}_{2}}\left(\bar{g}_{1}+\bar{g}_{2}\right) & =\mathcal{L}_{\boldsymbol{X}_{1}} \bar{g}_{1}+\mathcal{L}_{\boldsymbol{X}_{1}} \bar{g}_{2}+\mathcal{L}_{\boldsymbol{X}_{2}} \bar{g}_{1}+\mathcal{L}_{\boldsymbol{X}_{2}} \bar{g}_{2} \\
& =\mathcal{L}_{\boldsymbol{X}_{1}} \bar{g}_{1}+\mathcal{L}_{\boldsymbol{X}_{2}} \bar{g}_{2} .
\end{aligned}
$$

Note that (for $i=1,2) \mathcal{L}_{\boldsymbol{X}_{i}} \bar{g}_{i}$ is the trivial extension to the product of a tensor on $\bar{M}_{i}$. Thus we have $\mathcal{L}_{\boldsymbol{X}_{1}+\boldsymbol{X}_{2}}\left(\bar{g}_{1}+\bar{g}_{2}\right)=\alpha\left(\bar{g}_{1}+\bar{g}_{2}\right)$, if and only if $\mathcal{L}_{\boldsymbol{X}_{1}} \bar{g}_{1}=\alpha \bar{g}_{1}$ and $\mathcal{L}_{\boldsymbol{X}_{2}} \bar{g}_{2}=\alpha \bar{g}_{2}$.

On functions the Lie and exterior derivative agree and so by almost the same argument we have.

Lemma 3.6. Given pseudo-Riemannian manifolds $\left(\bar{M}_{1}, \bar{g}_{1}\right)$ and $\left(\bar{M}_{2}, \bar{g}_{2}\right)$, $\boldsymbol{X}_{1}$ is a gradient vector field on $\bar{M}_{1}$ and $\boldsymbol{X}_{2}$ is a gradient vector field on $\bar{M}_{2}$ if and only if $\boldsymbol{X}_{1}+\boldsymbol{X}_{2}$ is a gradient vector field on $\left(\bar{M}_{1} \times \bar{M}_{2}, \bar{g}_{1} \times \bar{g}_{2}\right)$.

Proof: Since, for $i=1,2$, the vector fields $\boldsymbol{X}_{i}$ are tangential to the leaf submanifolds, we have

$$
\left(\bar{g}_{1}+\bar{g}_{2}\right)\left(\boldsymbol{X}_{1}+\boldsymbol{X}_{2}, \quad\right)=\bar{g}_{1}\left(\boldsymbol{X}_{1},\right)+\bar{g}_{2}\left(\boldsymbol{X}_{2},\right) .
$$

If (for $i=1,2) \bar{g}_{i}\left(\boldsymbol{X}_{i},\right)=d_{i} f_{i}$ then summing shows that the left-hand side is $d\left(f_{1}+f_{2}\right)$. (Here $d_{i}$ denotes the exterior derivative on the factor manifolds which may be identified with the restriction of the exterior derivative $d$ on $\bar{M}_{1} \times \bar{M}_{2}$.) On the other hand if we have $\left(\bar{g}_{1}+\bar{g}_{2}\right)\left(\boldsymbol{X}_{1}+\boldsymbol{X}_{2}, \quad\right)=d f$, for some function $f$ on $\bar{M}_{1} \times \bar{M}_{2}$ then by restriction we obtain that $\bar{g}_{i}\left(\boldsymbol{X}_{i},\right)=$ $d_{i} f$ which shows that, on any leaf of the product, $\boldsymbol{X}_{i}$ is a gradient field. So $\boldsymbol{X}_{i}$ is a gradient on $\bar{M}_{i}$.

Recall, from the proof of Theorem [2.1, that on the ambient manifold there is a canonical Euler vector field $\boldsymbol{X}$. This is the Euler field from the $\mathbb{R}_{+}$-action on the cone $\mathcal{Q}$ and the trivial extension of this action via the product $\mathcal{Q} \times I=\tilde{M}$. From the formula (11) for the metric we see that $\boldsymbol{X}$ is a homothetic gradient field. This has an obvious origin in the case of a metric cone product construction, as follows.

Proposition 3.7. In the case that the ambient metric is a product of cone metrics, as in Proposition 3.1, then the canonical ambient Euler vector field $\boldsymbol{X}$ is the sum of the Euler fields for the metric cones $\bar{M}_{1}$ and $\bar{M}_{2}$.

Proof: Suppose that the ambient space $(\tilde{M}, \boldsymbol{h})$ is a product $\left(\bar{M}_{1} \times \bar{M}_{2}, \bar{g}_{1} \times\right.$ $\left.\bar{g}_{2}\right)$, as in Proposition 3.1, Each metric cone $\left(\bar{M}_{i}, \bar{g}_{i}\right)(i=1,2)$ has an Euler field $\boldsymbol{X}_{i}$ which is a 2-homothetic gradient field. Thus from the previous Lemmas $\boldsymbol{X}_{1}+\boldsymbol{X}_{2}$ is a 2-homothetic gradient field on $\tilde{M}$.

In terms of the coordinates used for the ambient metric in expression (11) the ambient Euler field is $t \partial / \partial t$. Using (91) and (10) this is easily re-expressed in terms of the cone coordinates $s_{1}$ and $s_{2}$ :

$$
\begin{aligned}
t \partial / \partial t & =t\left(\partial s_{1} \partial t \partial / \partial s_{1}+\partial s_{2} \partial t \partial / \partial s_{2}\right) \\
& =\frac{1}{2}\left(s_{1}+s_{2}\right)\left((1+\mu \rho) \partial / \partial s_{1}+(1-\mu \rho) \partial / \partial s_{2}\right) \\
& =s_{1} \partial / \partial s_{1}+s_{1} \partial / \partial s_{1} .
\end{aligned}
$$

Thus $\boldsymbol{X}=\boldsymbol{X}_{1}+\boldsymbol{X}_{2}$. 
Remark: Note that using Lemma 3.5. Lemma 3.6 and the formula (8) one can see immediately that the metric $\boldsymbol{h}_{\times}$is a Ricci-flat ambient metric without performing coordinate transformations to put it in form of (1): Writing $\boldsymbol{X}_{i}(i=1,2)$ for the respective cone Euler fields, $\boldsymbol{X}_{1}+\boldsymbol{X}_{2}$ is a 2-homothetic gradient field for $\boldsymbol{h}_{\times}$(and so property (i) of the ambient metric definition is satisfied). Along the hypersurface $s_{1}=s_{2}, \boldsymbol{h}_{\times}$obviously restricts to the tautological bilinear form for the conformal structure $\mathcal{Q} \rightarrow$ $\left(M_{1} \times M_{2}\right)$ (and so property (ii) of the ambient metric definition is satisfied). As mentioned earlier it is a product of Ricci-flat metrics and therefore Ricciflat.

We should point that, there is nevertheless considerable value in the normal form (11) for the ambient metric. This form has a very useful geometric interpretation, as outlined in [12]. For the purposes of this article, it enabled an extension of the cone product metric to a larger manifold. It also is valid for the case that the boundary structure is conformal to a product of Ricciflat metrics (a case for which the the metric cones are unavailable). Finally giving the ambient metric in this form yields immediate contact with the previous explicit treatments of the ambient manifold such as [12, 20] (where this nomalisation of the ambient metric is also used).

Finally, for later use, we observe that metric cones are characterised as follows [15].

Lemma 3.8. Let $(M, g)$ be a Ricci-flat pseudo-Riemannian space of signature $(p+1, q+1)$ admitting a homothetic gradient vector field $V$, i.e., $\nabla_{Z}^{g} V=c \cdot Z$ for all $Z \in T M$ and some constant $c \neq 0$.

(1) If $V$ is everywhere spacelike then $(M, g)$ is an open subset of the cone $(\bar{N}, \bar{h})$ defined over some Einstein space $(N, h)$ of positive scalar curvature with signature $(p, q+1)$.

(2) If $V$ is everywhere timelike then $(M, g)$ is an open subset of the cone $(\bar{N}, \bar{h})$ of some Einstein space $(N, h)$ of negative scalar curvature with signature $(p+1, q)$.

\section{The Poincaré Metric}

Suppose for a conformal $n$-manifold $M$ that there is an ambient metric $\boldsymbol{h}$

$$
\boldsymbol{h}:=2 t d t d \rho+2 \rho d t^{2}+t^{2} \tilde{g}(x, \rho)_{i j} d x^{i} d x^{j},
$$

where $\rho$ is a defining function for $\mathcal{Q}$ in the ambient manifold $\tilde{M}, t$ is homogeneous of degree 1 with respect to dilations on $\tilde{M}$ and the $x^{i}$ arise from coordinates on $M$. Here $\tilde{g}(x, \rho)$ is the pull-back to $\tilde{M}$ of a family of metrics on $M$ parametrised by $\rho$ and such that $g=g(x, 0)$ is a metric from the conformal class on $M$. Following [12] (see also [25]) we define $\left(M^{+}, g^{+}\right)$to be the embedded (hypersurface) structure given by the zero set of the defining function $\boldsymbol{h}(\boldsymbol{X}, \boldsymbol{X})+1$, with $g^{+}$the pull-back of $\tilde{g}$ to this embedded manifold. To study this explicitly we introduce new coordinates (on the $\rho<0$ side of $\mathcal{Q})$ in $\tilde{M}$ as follows. Let $r=\sqrt{-2 \rho}$ and $u=r t$. Then a direct calculation yields

$$
\boldsymbol{h}=u^{2} g^{+}-d u^{2}
$$


where

$$
g^{+}=r^{-2}\left(d r^{2}+g(x, r)_{i j} d x^{i} d x^{j}\right),
$$

with $g(x, r)_{i j}=\tilde{g}(x, \rho(r))_{i j}$. From (13) we see that the ambient structure is a metric cone manifold over $\left(M^{+}, g^{+}\right)$. The ambient Euler field $\boldsymbol{X}$ is calculated to be $\boldsymbol{X}=u \partial / \partial u$ in the new cone coordinates, and so over $\left(M^{+}, g^{+}\right)$it has the interpretation of the Euler field for this cone. From (6) it follows that if $\boldsymbol{h}$ is Ricci-flat (which we henceforth assume) then $g^{+}$ is Einstein with $\operatorname{Ric}\left(g^{+}\right)=-n g^{+}$. On the other hand from (14) (and since $r(\rho)$ extends smoothly to $\rho=0) g^{+}$has conformal infinity $(M,[g])$.

In particular we may apply this to the ambient metric (1) from Theorem 2.1. To respect that $(M, g)=\left(M_{1} \times M_{2}, g_{1} \times g_{2}\right)$, in that case we write $\left(M^{1,2}, g^{1,2}\right)$ for $\left(M^{+}, g^{+}\right)$and have the following result.

Theorem 4.1. To each pair of Einstein manifolds $\left(M_{1}^{m_{1}}, g_{1}\right)$ and $\left(M_{2}^{m_{2}}, g_{2}\right)$, $\left(m_{1} \geq 1, m_{2} \geq 0\right)$ satisfying $m_{2}\left(m_{2}-1\right) \operatorname{Sc}\left(g_{1}\right)=-m_{1}\left(m_{1}-1\right) \operatorname{Sc}\left(g_{2}\right)$, there is a conformally compact Einstein manifold $\left(M^{1,2}, g^{1,2}\right)$, with

$$
\operatorname{Ric}\left(g^{1,2}\right)=-\left(m_{1}+m_{2}\right) g^{1,2},
$$

and conformal infinity $\left(M_{1} \times M_{2},\left[g_{1} \times g_{2}\right]\right)$. This is given explicitly by

$$
M^{1,2}=M_{1} \times M_{2} \times I
$$

where, with $\mu$ satisfying $2 m_{1}\left(m_{1}-1\right) \mu:=\operatorname{Sc}\left(g_{1}\right)$ and $2 m_{2}\left(m_{2}-1\right) \mu:=$ $-\operatorname{Sc}\left(g_{2}\right)$, we have

$$
I= \begin{cases}{[0, \infty)} & \text { if } \mu=0, \\ {[0, \infty)} & \text { if } \mu<0 \text { and } m_{2}=0 \\ {[0, \infty) \backslash\left\{\sqrt{\frac{2}{\mu}}\right\}} & \text { if } \mu>0 \text { and } m_{2}=0 \\ {[0, \infty) \backslash\left\{\sqrt{\frac{2}{\mid \mu}}\right\}} & \text { otherwise. }\end{cases}
$$

and

$$
g^{1,2}=r^{-2}\left(d r^{2}+\left(1-\mu r^{2} / 2\right)^{2} g_{1}+\left(1+\mu r^{2} / 2\right)^{2} g_{2}\right) .
$$

Note that in the special cases that $m_{1}, m_{2} \leq 1$ there is a family of Poincaré metrics parametrised by $\mu$.

\section{The ambient metric Over a Poincaré-Einstein metric}

We digress briefly to observe here that the above construction of the Poincaré-Einstein metric is reversible, and this gives a notion of an ambient metric over any Poincaré-Einstein metric. We recover the ambient metric as a simple extension of the metric cone over the interior (or bulk) of the Poincaré metric structure. We will need this result in section 7 , For simplicity of exposition we will assume that the Poincaré-Einstein structure is smooth, however the construction extends in an obvious way to metrics with some specified regularity.

Suppose that $\left(\underline{M}^{n+1}, \underline{g}, r\right)$ is a Poincaré-Einstein structure. That is $\underline{M}$ is a manifold with boundary a smooth manifold $\partial \underline{M}=M, r$ is a non-negative defining function for $M$, and, off the boundary $g^{+}:=r^{-2} g$ is Einstein with scalar curvature $-n(n+1)$. Then, as mentioned above, the restriction of $\underline{g}$ to 
$T M$ in $\left.T M^{+}\right|_{M}$ determines a conformal structure $[g]$. We define the ambient manifold over $\left(\underline{M}^{n+1}, \underline{g}, r\right)$ to be $\tilde{M}=\underline{M} \times \mathbb{R}_{+}$. We write $\pi: \tilde{M} \rightarrow \underline{M}$ for the projection $\tilde{M} \ni(p, u) \mapsto p \in \underline{M}$ and $\mathcal{Q}:=\pi^{-1}(M)$.

The manifold $\tilde{M}$ is equipped with a metric and smooth structure as follows. Off the boundary we use the usual product smooth structure on $M^{+} \times \mathbb{R}_{+}$. We will use $u$ here for the standard coordinate on $\mathbb{R}_{+}$. The defining function determines, for some $\epsilon>0$, an identification of $M \times[0, \epsilon)$ with a neighbourhood of $M$ in $\underline{M}$ : since $|d r|_{g}$ is non-vanishing along the boundary, $(p, y) \in M \times[0, \epsilon)$ is identified with the point obtained by following the flow of the gradient $\bar{g}^{-1}(d r$,$) , through p$, for $y$ units of time. Thus over this we also have an identification of $\tilde{M}$ with $M \times[0, \epsilon) \times \mathbb{R}_{+}$. Suppose that $x^{i}$ are local coordinates on $U \subset M$ then on $U \times(0, \epsilon) \times \mathbb{R}_{+}$we have coordinates $\left(x^{i}, r, u\right)$. We construct a coordinate patch for $\tilde{M}$ over $U \times[0, \epsilon)$ by taking coordinates $\left(x^{i}, \rho, t\right)$ on $U \times\left(-\epsilon^{2} / 2,0\right] \times \mathbb{R}_{+}$and identifying this space with $\pi^{-1}(U \times[0, \epsilon))$ by the coordinate transformation $\rho=-\frac{1}{2} r^{2}, t=u / r$ on $\pi^{-1}(U \times(0, \epsilon))$. This is obviously independent of the coordinates $x^{i}$, local on $M$. Thus, by doing this for all coordinate patches on $M$, this extends a smooth structure to $\tilde{M}$.

We take the cone metric $h:=u^{2} g^{+}-d u^{2}$ on $\pi^{-1}\left(M^{+}\right)$. This cone metric is Ricci flat and so it remains to verify that it extends to a non-degenerate metric on $\mathcal{Q}$.

The condition $\operatorname{Ric}\left(g^{+}\right)=-n g^{+}$implies that on $M|d r|_{g}=1$. However a choice of metric $g$ (from the conformal class) on $M$ determines a unique defining function $r$, in a neighbourhood of $M$, by requiring $|d r|_{g}=1$ and $\left.g\right|_{T M}=g$. The defining function determines, for some $\epsilon>0$, an identification of $M \times[0, \epsilon)$ with a neighbourhood of $M$ in $\underline{M}$ and in terms of this the metric $g^{+}$takes the form

$$
g^{+}=r^{-2}\left(g_{r}+d r^{2}\right)
$$

where $g_{r}$ is a 1-parameter family of metrics on $M$. See [23, 20 for details (in the case of Riemannian signature but the argument there is essentially unaltered for other signatures given our assumptions). The change from a general defining function to one that satisfies $|d r|_{g}=1$ in a neighbourhood of $M$ is achieved by a smooth rescaling $r \mapsto e^{\omega} r$ (for some smooth function $\omega$ ) thus assuming that we have such a normalised $r$ does not affect the smooth structure on $\tilde{M}$. Using (15) and the coordinate transformation $\rho=-\frac{1}{2} r^{2}$, $t=u / r$ on $\pi^{-1}(U \times(0, \epsilon))$ it follows easily that the metric $\boldsymbol{h}$ may be written in the form (2) and so obviously extends as a metric to $\mathcal{Q}$. Note that the coordinate change $\rho=-r^{2} / 2$ means the ambient metric is not smooth at the boundary in general. In fact from the Einstein condition it follows that the Taylor series of $g_{r}$ involves only even powers of $r$ up to the $r^{n}$ term, and so the ambient metric is differentiable to any order less than $n / 2[23]$.

Remark: There is an obvious variant of the above construction where one would only assume the Poincaré metric is asymptotically Einstein. In this case the ambient metric will be asymptotically Ricci-flat. 


\section{HolONOMY}

First we observe a general result. Let $(M, g)$ be a pseudo-Riemmanian signature $(p, q)$-manifold. We write $\mathcal{F}_{q}$ to denote a frame based at $q \in M$ and $A \cdot \mathcal{F}_{q}$ for the obvious action of $A \in \mathrm{O}(p, q)$ acting on $\mathcal{F}_{q}$. If $\gamma_{q}$ is the trace of a closed curve based at $q$ then we write $\mathcal{F}_{q}^{\gamma_{q}}$ for the frame obtained from $\mathcal{F}_{q}$ by parallel translation around $\gamma_{q}$. Recall that the holonomy, based at $q$, of the metric $g$ is by definition the group

$$
\operatorname{Hol}_{q}(M, g)=\left\{A \in \mathrm{O}(p, q) \text { : for any frame } \mathcal{F}_{q} \exists \gamma_{q} \text { s.t. } \mathcal{F}_{q}^{\gamma_{q}}=A \cdot \mathcal{F}_{q}\right\}
$$

Now suppose that on $M$ there is everywhere a homothetic gradient field $v$. That is a constant $c$ such that

$$
\mathcal{L}_{v} g=c g
$$

or equivalently $\nabla_{u} v=\frac{c}{2} u$ for all $u \in \Gamma(T M)$. Let us say a hypersurface $E$ in $M$ is $v$-transverse if each maximal integral curve of $v$ meets $E$ in exactly one point. In this setting the holonomy of $g$ is recovered from curves $\gamma^{E}$ in the $v$-transverse submanifold. More precisely we have the following.

Theorem 6.1. Let $(M, g)$ be a pseudo-Riemmanian signature $(p, q)$-manifold with a nowhere-vanishing homothetic gradient field $v$ and a $v$-transverse hypersurface $E$. Then for $q \in E$

$\operatorname{Hol}_{q}(M, g)=\left\{A \in \mathrm{O}(p, q)\right.$ : for any frame $\mathcal{F}_{q} \exists \gamma_{q}^{E} \subset E$ s.t. $\left.\mathcal{F}_{q}^{\gamma_{q}^{E}}=A \cdot \mathcal{F}_{q}\right\}$

We need some preliminary notation and results before we prove the Theorem. Let us parametrise the integral curves of $v$ by a smooth function $s$ on $M$ which vanishes on $E$. Write $\varphi_{p, s}$ for the flow of $v$ through $p \in M$ at a time $s$. For $q \in M$ let us fix attention on a closed smooth path $\gamma_{q}:[0,1] \rightarrow M$, $\gamma_{q}(0)=\gamma_{q}(1)=q$. Over $\gamma_{q}$ we construct a 2-parameter path-cone:

$$
\Gamma_{q}: D \subset[0,1] \times \mathbb{R} \rightarrow M
$$

given by

$$
\Gamma_{q}(t, s):=\varphi_{\gamma_{q}(t), s} \circ \gamma_{q}(t),
$$

where $D$ is the open subset of $[0,1] \times \mathbb{R}$ which gives the maximal range of definition of the flow ( $s$ runs over an interval that depends on $t$ ). By analogy with our treatment of curves we will also use $\Gamma_{q}$ to denote the trace (graph) of this function in $M$ since in any instance the meaning should be clear by context. Now for $F_{q} \in T_{q} M$ we write $F_{q}(t)$ for the field (at time $t$ ) along the trace of $\gamma_{q}(t)$ given by parallel translation of $F_{q}$. We extend this to the path cone by parallel translation along the flow lines of $v$; we write $F_{q}(t, s)$ for the vector in $T_{\Gamma_{q}(t, s)} M$ given by the parallel transport of $F_{\gamma_{q}(t)}$ to $\varphi_{\gamma_{q}(t), s}$ along the integral curve of $v$ through $\gamma_{q}(t)$. We need to compare parallel transport in this way with Lie dragging.

Lemma 6.2.

$$
F_{q}(t, s)=\left(1-\frac{c}{2} s\right) \varphi_{\gamma_{q}(t), s *}\left(F_{q}(t)\right) .
$$

Proof: Since the Levi-Civita connection $\nabla$ is torsion free we have

$$
\nabla_{v_{s}} F_{q}(t, s)=\nabla_{F_{q}(t, s)} v+\mathcal{L}_{v} F_{q}(t, s) .
$$


This vanishes since by construction $F_{q}(t, s)$ is parallel along the flow lines of $v$. On the other hand, since $v$ is homothetic we have $\nabla_{F_{q}(t, s)} v=\frac{c}{2} F_{q}(t, s)$ so

$$
\mathcal{L}_{v} F_{q}(t, s)=-\frac{c}{2} F_{q}(t, s) .
$$

This sets us up for the key result on the path-cone which is as follows.

Lemma 6.3. The field $F_{q}(t, s)$ is parallel along $\Gamma_{q}$.

Proof: Let us write $\dot{\gamma}_{q}^{s}(t)$ for a tangent field to the curve

$$
\Gamma_{q}(, s):[0,1] \rightarrow M
$$

determined by a fixed value of $s$. Now it follows from the $s$ derivative of $\Gamma_{q}(t, s)$ that $\dot{\gamma}_{q}^{s}(t)=\varphi_{\gamma_{q}(t), s *}\left(\dot{\gamma}_{q}^{s}(t)\right)$. This with the previous lemma implies

$$
\nabla_{\dot{\gamma}_{q}^{s}(t)} F_{q}(t, s)=\left(1-\frac{c}{2} s\right) \nabla_{\varphi_{\gamma_{q}(t), s *\left(\dot{\gamma}_{q}^{s}(t)\right)}} \varphi_{\gamma_{q}(t), s *}\left(F_{q}(t)\right),
$$

since $s$ is constant. But since $v$ is a homothetic gradient field its flow preserves the Levi-Civita connection. So

$$
\nabla_{\varphi_{\gamma_{q}(t), s *\left(\dot{\gamma}_{q}^{s}(t)\right)}} \varphi_{\gamma_{q}(t), s *}\left(F_{q}(t)\right)=\varphi_{\gamma_{q}(t), s *}\left(\nabla_{\left(\dot{\gamma}_{q}^{s}(t)\right)} F_{q}(t)\right)=0 .
$$

So at each point of $\Gamma_{q}, F_{q}(t, s)$ is parallel in the direction $\dot{\gamma}_{q}^{s}(t)$. But it is also parallel in the direction of $v$ and so the result follows.

Proof of the Theorem: First observe that since each integral curve of $v$ meets the $v$-transverse hypersurface in exactly one point, there is a canonical smooth projection $\pi: M \rightarrow E$ which for $p \in M$ finds the point $\pi(p) \in E$ on the flow through $p$. Thus given an arbitrary closed path $\gamma_{q}$ (based at $q \in E$ ) there is a path $\gamma_{q}^{E}$ with trace in $E$ given by $\gamma_{q}^{E}(t)=\pi \circ \gamma_{q}(t)$. This determines a function $S_{E}(t)$ which gives the value of the parameter $s$ where $E$ meets $\Gamma_{q}$. That is $\gamma_{q}^{E}(t)=\Gamma_{q}\left(t, s_{E}(t)\right)$. There is a vector field along the trace of $\gamma^{E}$

$$
F_{q}^{E}(t):=F_{q}\left(t, s_{E}(t)\right) .
$$

From the last Lemma this is parallelly transported around $\gamma^{E}$ :

$$
\nabla_{\dot{\gamma}_{q}^{E}(t)} F_{q}^{E}(t)=0
$$

By construction $F_{q}(1)=F_{q}^{E}(1)$, since $s_{E}(1)=0$. Since this holds for all vectors $F_{q} \in T_{q} M$ and for all closed paths $\gamma_{q}$ the proof is complete.

Theorem 6.4. The holonomy group of the $\mathcal{Q}$ connected component of the ambient manifold, with metric (1), is the same as the conformal holonomy of the underlying conformal manifold $\left(M_{1} \times M_{2},\left[g_{1} \times g_{2}\right]\right)$.

Proof: We will retain only the $\mathcal{Q}$ connected component of the ambient manifold and term this the ambient manifold.

First we treat the case that $\operatorname{Sc}\left(g_{1}\right)=m_{1}\left(m_{1}-1\right) \lambda$ for $\lambda \neq 0$. Then $(\tilde{M}, \boldsymbol{h})$ is a product of cones $\bar{M}_{1}$ and $\bar{M}_{2}$ with metrics given, respectively, as in (7). The holonomy of the product, the ambient manifold, is the product of the component holonomy groups. On each cone there is a homothetic gradient field in the sense of Theorem 6.1 above. These are respectively $s_{1} \partial / \partial s_{1}$ and $s_{2} \partial / \partial s_{2}$. Thus on each cone $\bar{M}_{i}(i=1,2)$ the holonomy may be computed by 
considering paths only in the $s_{i}=1$ transverse hypersurface. It follows easily that the ambient holonomy is generated by (the transport of full frames for $T\left(\bar{M}_{1} \times \bar{M}_{2}\right)$ along) loops in the codimension 2 submanifold $s_{1}=1=s_{2}$. But this submanifold is in $\mathcal{Q}$ and is a section over $M_{1} \times M_{2}$ and so this holonomy group is exactly the conformal holonomy, that is holonomy of the normal tractor connection. To see this last claim we use following result of [9] (see also [18]). Write $\left.T \tilde{M}\right|_{\mathcal{Q}}$ for the restriction of the ambient tangent bundle to $\mathcal{Q}$ and define an action of $\mathbb{R}_{+}$on this space by $s^{-1} \sigma_{*}^{s} \cdot \xi$. Here $\sigma$ is the principal action of $\mathbb{R}_{+}$on $\mathcal{Q}$ given by $\sigma^{s}\left(g_{x}\right)=s^{2} g_{x}$. Then the quotient $\left(\left.T \tilde{M}\right|_{\mathcal{Q}}\right) / \mathbb{R}_{+}$is a vector bundle over $\mathcal{Q} / \mathbb{R}_{+}=M$. This may be identified with the standard conformal tractor bundle and via this identification the ambient parallel transport induces the normal tractor connection. It follows immediately that parallel transport along any fixed section of $\mathcal{Q}$ is sufficient to recover the conformal holonomy.

Now in the case of $\lambda=0$ suppose the ambient metric is given by (11). Then $\partial / \partial \rho$ is parallel, and hence a homothetic gradient field. This is obviously transverse to $\mathcal{Q}$ and so the ambient holonomy may be calculated by paths in $\mathcal{Q}$. From the result that the $\mathbb{R}_{+}$-action on $\left(\left.T \tilde{M}\right|_{\mathcal{Q}}\right)$ by $\xi \mapsto-1 \sigma_{*}^{s} \cdot \xi$ agrees with parallel transport 9, 18] it follows easily that the ambient holonomy may be calculated via loops in a section of $\mathcal{Q}$ and thus agrees with the conformal holonomy.

\section{Characterisation by special Killing forms}

We want to characterise here sub-product spaces $\left(M^{1,2}, g^{1,2}\right)$ (as they were constructed in Theorem 4.1) by the existence of certain differential forms subject to a system of so-called special Killing form equations.

To start with, let

$$
g^{1,2}=r^{-2}\left(d r^{2}+\left(1-\mu r^{2} / 2\right)^{2} g_{1}+\left(1+\mu r^{2} / 2\right)^{2} g_{2}\right)
$$

be a Poincaré-Einstein metric on $M^{1,2}=M_{1} \times M_{2} \times I$ as in Theorem 4.1. We assume here that the factors $M_{1}^{m_{1}}$ and $M_{2}^{m_{2}}$ of the sub-product are oriented spaces of dimensions $m_{1} \geq 1$ and $m_{2} \geq 0$. Then we denote with $\operatorname{vol}\left(g_{i}\right)$, $i=1,2$, the volume forms, which correspond to the metrics $g_{1}$ resp. $g_{2}$. We use the same notation for the pull-backs of these volume forms to the sub-product $M^{1,2}$.

Lemma 7.1. Let $\mu>0$. The $m_{1}$-form

$$
\psi:=\left(\frac{\mu r}{2}-\frac{1}{r}\right)^{m_{1}+1} \cdot \operatorname{vol}\left(g_{1}\right)
$$

on $\left(M^{1,2}, g^{1,2}\right)$ satisfies the differential equations

$$
\nabla^{1,2} \psi=\frac{1}{m_{1}+1} d \psi \quad \text { and } \quad \nabla_{Y}^{1,2} d \psi=\left(m_{1}+1\right) \cdot g^{1,2}(Y, \cdot) \wedge \psi
$$

for all $Y \in T M^{1,2}$, where $\nabla^{1,2}$ denotes the Levi-Civita connection of $g^{1,2}$. The function $r_{\#}:=\left(|\psi|_{g^{1,2}}^{2}\right)^{-1 / 2}$ is a defining function with $\left|d r_{\#}\right|_{\partial M^{1,2}}=1$ for the conformal boundary $\partial M^{1,2}:=M_{1} \times M_{2}$. 
Proof. We use the coordinate change $s=\ln \left(\sqrt{\frac{\mu}{2}} \cdot r\right)$. Then we set $h_{1}:=$ $\sqrt{2 \mu} \cdot \sinh (s)=\left(\frac{\mu r}{2}-\frac{1}{r}\right)$ and $h_{2}:=\sqrt{2 \mu} \cdot \cosh (s)$ and the metric $g^{1,2}$ takes the form

$$
d s^{2}+2 \mu\left(\sinh ^{2}(s) \cdot g_{1}+\cosh ^{2}(s) \cdot g_{2}\right) .
$$

Let $\left\{e_{1}, \ldots, e_{m_{1}}\right\}$ denote a local orthonormal frame for $g_{1}$ on $M_{1}$ and let $\left\{f_{1}, \ldots, f_{m_{2}}\right\}$ be a local orthonormal frame on $\left(M_{2}, g_{2}\right)$. We set $g_{i j}^{1}:=$ $g_{1}\left(e_{i}, e_{j}\right)$ for $i, j \in\left\{1, \ldots, m_{1}\right\}$ and $g_{i j}^{2}:=g_{2}\left(f_{i}, f_{j}\right)$ for $i, j \in\left\{1, \ldots, m_{2}\right\}$. Moreover, we denote by $e_{i}^{b}$ and $f_{i}^{b}$ the dual 1 -forms with respect to $g_{1}$ resp. $g_{2}$. The pull-back of these gives rise to a local (orthogonal) coframe $\left\{d s, e_{1}^{b}, \ldots, e_{m_{1}}^{b}, f_{1}^{b}, \ldots, f_{m_{2}}^{b}\right\}$ on $M^{1,2}$. Locally, it holds that $\psi=h_{1}^{m_{1}+1} \cdot e_{1}^{b} \wedge$ $\cdots \wedge e_{m_{1}}^{b}$. For the covariant derivatives we find the formulae (cf. [34])

$$
\begin{array}{llrl}
\nabla_{\frac{\partial}{\partial s}}^{1,2} e_{i}^{b}=-\frac{h_{1}^{\prime}}{h_{1}} \cdot e_{i}^{b}, & \nabla_{\frac{\partial}{\partial s}}^{1,2} f_{i}^{b}=-\frac{h_{2}^{\prime}}{h_{2}} \cdot f_{i}^{b}, & \nabla_{\frac{\partial}{\partial s}}^{1,2} d s=0, \\
\nabla_{e_{j}}^{1,2} e_{i}^{b}=\nabla_{e_{j}}^{g_{1}} e_{i}^{b}-\frac{h_{1}^{\prime}}{h_{1}} g_{j i}^{1} d s, & \nabla_{e_{j}}^{1,2} f_{i}^{b}=0, & \nabla_{e_{j}}^{1,2} d s=h_{1}^{\prime} h_{1} \cdot e_{j}^{b}, \\
\nabla_{f_{j}}^{1,2} f_{i}^{b}=\nabla_{e_{j}}^{g_{2}} e_{i}^{b}-\frac{h_{2}^{\prime}}{h_{2}} g_{j i}^{2} d s, & \nabla_{f_{j}}^{1,2} e_{i}^{b}=0, & \nabla_{f_{j}}^{1,2} d s=h_{2}^{\prime} h_{2} \cdot f_{j}^{b},
\end{array}
$$

where $h_{i}^{\prime}:=\frac{\partial}{\partial s} h_{i}$ and $\nabla^{g_{i}}, i=1,2$, denote the Levi-Civita connections of $g_{1}$ resp. $g_{2}$. Further, we obtain

$$
\nabla_{e_{i}}^{1,2} \psi=-g_{i i}^{1} h_{1}^{\prime} h_{1}^{m_{1}} \cdot e_{1}^{b} \wedge \cdots \wedge d s \wedge \cdots \wedge e_{m_{1}}^{b}=(-1)^{i} g_{i i}^{1} \frac{h_{1}^{\prime}}{h_{1}} \cdot d s \wedge\left(\iota_{e_{i}} \psi\right)
$$

(where in the middle part of the equation $d s$ replaces $e_{i}$ at the $i$-th position of the $\wedge$-product)

$$
\nabla_{\frac{\partial}{\partial s}}^{1,2} \psi=\frac{h_{1}^{\prime}}{h_{1}} \psi \quad \text { and } \quad \nabla_{f_{i}}^{1,2} \psi=0
$$

This implies $d \psi=\left(m_{1}+1\right) \cdot \frac{h_{1}^{\prime}}{h_{1}} d s \wedge \psi$, which shows that $\psi$ is a Killing form, i.e., $\nabla^{1,2} \psi=\frac{1}{m_{1}+1} d \psi$. Moreover, we calculate

$$
\begin{aligned}
& \nabla_{\frac{\partial}{\partial s}}^{1,2} d \psi=\left(m_{1}+1\right) \cdot \frac{h_{1}^{\prime \prime}}{h_{1}} d s \wedge \psi=\left(m_{1}+1\right) \cdot g^{1,2}\left(\frac{\partial}{\partial s}, \cdot\right) \wedge \psi \\
& \nabla_{f_{i}}^{1,2} d \psi=\left(m_{1}+1\right) \cdot \frac{h_{1}^{\prime} h_{2}^{\prime} h_{2}}{h_{1}} f_{j}^{b} \wedge \psi=\left(m_{1}+1\right) \cdot g^{1,2}\left(f_{i}, \cdot\right) \wedge \psi \quad \text { and } \\
& \nabla_{e_{i}}^{1,2} d \psi=0 .
\end{aligned}
$$

The latter relations show that the second differential equation stated in Lemma 7.1 is also satisfied.

For the square length of $\psi$ with respect to $g^{1,2}$ we calculate $|\psi|^{2}=h_{1}^{2}$ and this implies that $r_{\#}=-h_{1}^{-1}$, which vanishes for $r \rightarrow 0$. Hence it is a defining function with $\left|d r_{\#}\right|=1$ on the conformal boundary $\partial M^{1,2}$.

We remark that the assumption $\mu>0$ in Lemma 7.1 does not cause any loss of generality as we do not make an assumption on the signature of the metric $g^{1,2}$. 
In general, a differential $p$-form $\varphi$ on some space $\left(\underline{N}^{n+1}, g\right)$ of dimension $n+1$, which satisfies

$$
\nabla^{g} \varphi=\frac{1}{p+1} d \varphi \quad \text { and } \quad \nabla_{Y}^{g} d \varphi=c \cdot g(Y, \cdot) \wedge \varphi
$$

for all $Y \in T \underline{N}$ and some constant $c \in \mathbb{R} \backslash\{0\}$, is called a special Killing form (cf. [35, 30]). The Killing constant is related to the scalar curvature of $g$ by $c=-\frac{(p+1) S c(g)}{(n+1) n}$. On an oriented space $\underline{N}$ it is straightforward to see that if $\varphi$ is special Killing then so is $\star d \varphi$, where $\star$ denotes the Hodge operator (cf. [30]). There is also a description for special Killings forms in terms of the cone. Let $\tilde{g}=-\operatorname{sgn}(c)\left(\frac{-c u^{2}}{p+1} g+d u^{2}\right)$ be the cone metric for $g$ on $\tilde{N}=\underline{N} \times \mathbb{R}_{+}$. (From the given relation of $c$ to the scalar curvature it is clear that this is in fact the cone as defined in section 3.) For the pull-back of $\varphi$ to $\tilde{N}$, we will also write $\varphi$. It is shown in [35] that the $(p+1)$-form

$$
\tilde{\varphi}=u^{p} d u \wedge \varphi+\frac{u^{p+1}}{p+1} d \varphi
$$

is parallel with respect to the Levi-Civita connection of the cone metric. On the other hand, if a cone metric $\tilde{g}$ of some $g$ admits a parallel $(p+1)$-form $\tilde{\varphi}$ then the $p$-form

$$
\varphi=\iota_{X} \tilde{\varphi}
$$

(restricted to the 1-level of the cone) is a special Killing form on $(\underline{N}, g)$. Here $\iota_{X}$ denotes the insertion of the Euler vector $X=u \partial / \partial u$.

We want to use this correspondence to prove a characterisation result for sub-products of Poincaré-Einstein metrics of the form $g^{1,2}$ (cf. Theorem 4.1). Let us call a differential form $\varphi$ non-degenerate and simple if it is at every point of $\underline{N}$ a $\wedge$-product of 1 -forms and has nowhere vanishing length. We denote by $\gamma^{\#}$ the dual vector to a 1 -form $\gamma$ with respect to $g$ on $\underline{N}$.

Theorem 7.2. Let $\left(\underline{N}^{n+1}, g\right)$ be a simply connected Poincaré-Einstein space of dimension $n+1$ with $\operatorname{Ric}(g)=-n g$ and conformal boundary $\underline{\partial \underline{N}}=M^{n}$.

(1) Suppose that there exists a non-degenerate and simple $m_{1}$-form $\psi$ which satisfies the differential equations

$$
\nabla^{g} \psi=\gamma \wedge \psi \quad \text { and } \quad\left(\nabla_{Y}^{g} \gamma\right) \wedge \psi=g(Y, \cdot) \wedge \psi
$$

for all $Y \in T \underline{N}$ on the bulk of $\underline{N}$, where $\gamma$ is a 1-form such that

$$
\iota_{\gamma} \psi=0 \quad \text { and } \quad g\left(\gamma^{\#}, \gamma^{\#}\right)>1
$$

then $(\underline{N}, g)$ is a sub-product as constructed in Theorem 4.1 with a metric on the bulk of the form

$$
r^{-2}\left(d r^{2}+\left(1-\mu r^{2} / 2\right)^{2} g_{1}+\left(1+\mu r^{2} / 2\right)^{2} g_{2}\right) .
$$

(2) If $\left(\underline{N}^{n+1}, g\right)$ is a sub-product as described in Theorem 4.1 then locally on the bulk of $\underline{N}$ there exists a $m_{1}$-form $\psi$ which satisfies the system of differential equations with respect to some 1-form $\gamma$ as in (1). Near the boundary $|\psi|^{-1}$ is a defining function.

Proof. First, let us assume that $g:=g^{1,2}$ on $\underline{N}=M_{1} \times M_{2} \times I$ is of the form as described in Theorem 4.1. Then we know from Lemma 7.1 that locally (with some choice of orientation) the non-degenerate and simple 
differential form $\psi=\left(\frac{\mu r}{2}-\frac{1}{r}\right)^{m_{1}+1} \cdot \operatorname{vol}\left(g_{1}\right)$ is special Killing. In particular, it holds that $\nabla^{g} \psi=\frac{h_{1}^{\prime}}{h_{1}} d s \wedge \psi$. We set $\gamma:=\frac{h_{1}^{\prime}}{h_{1}} d s$ and together with the formulae for the covariant derivative of $d s$ from the proof of Lemma 7.1 we see that the demanded conditions are satisfied for this choice of $\psi$. In particular, the 1-form $\gamma$ has length greater than 1. The length function $|\psi|_{g}$ tends to infinity near the boundary and its inverse $|\psi|_{g}^{-1}$ is locally a defining function (cf. Lemma 7.1).

On the other side, let $\psi$ be a differential form on $\underline{N}$ such that

$$
\nabla^{g} \psi=\gamma \wedge \psi \quad \text { and } \quad\left(\nabla_{Y}^{g} \gamma\right) \wedge \psi=g(Y, \cdot) \wedge \psi
$$

for all $Y \in T \underline{N}$, where $\gamma$ is some smooth 1-form with the properties as in (11). The first equation implies immediately that $\psi$ is a Killing form, i.e., $\nabla^{g} \psi=\frac{1}{m_{1}+1} d \psi$. The second condition implies $\nabla_{Y}^{g} d \psi=\left(m_{1}+1\right) \cdot g(Y, \cdot) \wedge \psi$, i.e., $\psi$ is special Killing.

We consider now the ambient metric $\tilde{g}$ on $\tilde{M}=\underline{N} \times \mathbb{R}_{+}$(with boundary as in section 5D) over the Poincaré-Einstein space $(\underline{N}, g)$. Over the bulk of $\underline{N}$ the ambient metric $\tilde{g}$ is just the cone metric $u^{2} g-d u^{2}$. The $\left(m_{1}+1\right)$-form $\tilde{\psi}=u^{m_{1}} d u \wedge \psi+\frac{u^{m_{1}+1}}{m_{1}+1} d \psi$ is parallel on this cone. With the assumptions on $\psi$ and $\gamma$, it follows that $\tilde{\psi}$ is non-degenerate and simple. Moreover, since $\underline{N}$ is simply connected, the ambient space $\tilde{M}$ itself is simply connected and orientable and we can apply a Hodge star operator on $\tilde{\psi}$ to obtain a non-degenerate and simple parallel differential form $\star \tilde{\psi}$. This shows by the deRham decomposition Theorem (cf. [36]) that $\tilde{g}$ is isometric to a product $\bar{g}_{1} \times \bar{g}_{2}$ of Ricci-flat metrics on some product space $\bar{M}_{1} \times \bar{M}_{2}$ (which includes $\tilde{M}$ as a submanifold with boundary).

Inserting the Euler vector $X=u \partial / \partial u$ into the parallel differential forms $\tilde{\psi}$ and $\star \tilde{\psi}$ reproduces the special Killing forms $\psi$ resp. $\star d \psi$ on $\underline{N}$. The latter form is equal to $\frac{1}{m_{1}+1} \star(\gamma \wedge \psi)$ and has by assumption no zeros on $\underline{N}$. This shows that the projections of the Euler vector $X$ to the factors $\bar{M}_{1}$ and $\bar{M}_{2}$ of the product structure on the ambient space have no singularities and are either everywhere timelike or spacelike. Since these projections of $X$ are homothetic gradient vector fields (cf. Lemma 3.5 and Lemma 3.6), we can conclude by using Lemma 3.8 that $\bar{g}_{1}$ and $\bar{g}_{2}$ are cone metrics over some Einstein spaces $\left(M_{1}, g_{1}\right)$ and $\left(M_{2}, g_{2}\right)$. By choosing appropriate scales for the metrics $g_{1}$ and $g_{2}$ it is straightforward to see that $g_{1} \times g_{2}$ is just a metric in the conformal class of the boundary $M=M_{1} \times M_{2}$ of the initial Poincaré-Einstein space $(\underline{N}, g)$. In particular, the ambient space $(\tilde{M}, \tilde{g})$ is a submanifold of the ambient space of $\left(M_{1} \times M_{2}, g_{1} \times g_{2}\right)$ that we introduced in Theorem 2.1. It follows that the initial Poincaré-Einstein space $(\underline{N}, g)$ is a sub-product space as constructed in Theorem 4.1.

\section{EXAmples AND MUlTiPle SUB-PRODUCTS}

It should be expected that, for Einstein manifolds, the generic situation is that there is a single Einstein metric in the conformal class (ignoring constant dilations of the metric). However to be concrete we give here 
some examples of Poincaré-Einstein metrics from Theorem 4.1 where the boundary conformal structure is not conformally Einstein.

Let us consider the special orthogonal group $S O(4)$ in dimension 4 . This is a 6-dimensional compact semisimple Lie group and the Killing form $B$ of the Lie algebra $s o(4)$ is an invariant non-degenerate negative definite symmetric biliniear form, which gives rise to a bi-invariant (negative definite) Riemannian metric $g_{B}$ on $S O(4)$. This metric is well-known to be Einstein of negative scalar curvature $-3 / 2$. In fact, the conformal holonomy algebra of the conformal class which is given by $g_{B}$ on $S O(4)$ is equal to $s o(7)$ (cf. [29]). The subalgebra $s o(7)$ sits naturally as subalgebra in the structure algebra so $(1,7)$ and acts trivially on a 1-dimensional subspace of the 8-dimensional standard representation $\mathbb{R}^{1,7}$. This shows that there exists (up to constants multiples) exactly one parallel standard tractor on $S O(4)$ (which, of course, corresponds to the Einstein metric $\left.g_{B}\right)$.

Now we define $M:=S O(4) \times S O(4)$ with metric $g_{B \times B}:=g_{B}^{1} \times g_{B}^{2}$, where $g_{B}^{1}$ is $-g_{B}$ on the first factor, and $g_{B}^{2}$ is $g_{B}$ on the second factor. This is a product of Einstein metrics, which satisfies the scalar curvature relation of Theorem 2.1 for the construction of a Ricci-flat ambient metric. By the unique existence (up to multiples) of Einstein scales on the factors and using Theorem 3.4 we know that $M$ with metric $g_{B \times B}$ is not conformally Einstein. The corresponding Poincaré-Einstein metric is explicitly given on $M \times\left[0, r_{o}\right)$ with $r_{0}=4 \cdot \sqrt{5}$ by

$$
r^{-2}\left(d r^{2}+\left(1-r^{2} / 80\right)^{2} g_{B}^{1}+\left(1+r^{2} / 80\right)^{2} g_{B}^{2}\right) .
$$

Another way to make examples is by using 4-manifolds in the sub-product construction. Einstein Riemannian 4-manifolds have only one Einstein scale, unless they are conformally flat. This is easily seen as follows. Suppose we have two linearly independent almost-Einstein structures on a 4-manifold. This exactly means that the manifold admits two linearly independent parallel standard tractors $I_{1}$ and $I_{2}$. The exterior product of these $I_{1} \wedge I_{2}$ is obviously parallel. This (adjoint) tractor $I_{1} \wedge I_{2}$ is a jet prolongation of a conformal gradient field $k$ which annihilates the Weyl curvature $C$ (i.e., $\iota_{k} C=0$ ), see section 2.3 of [17]. Since the parallel tractor $I_{1} \wedge I_{2}$ is a prolongation of $k$ and parallel it follows immediately that $k$ is non-vanishing on an open dense set in the manifold. On the other hand in dimension 4 we have the identity $|C|^{2} \delta_{b}^{a}=4 C^{a c d e} C_{b c d e}$, and so $|C|^{2}=0$ on an open dense set, and hence everywhere.

Finally, we present a recursive construction principle of multiple subproducts based on Theorem 4.11in order to produce Poincaré-Einstein spaces. For this purpose we set up the following initial data. Let $\left(M_{0}^{m_{0}}, g_{0}\right)$ be an Einstein space of negative scalar curvature $S c\left(g_{0}\right)=-m_{0}\left(m_{0}-1\right)$ with $\operatorname{dim}\left(M_{0}\right)=m_{0}$, i.e., $\mu=-1 / 2$. Further, let $\ell \geq 1$ be a positive integer and let $\left(M_{i}^{m_{i}}, g_{i}\right), i \in\{1, \ldots, \ell\}$, be Einstein spaces with positive scalar curvature $S c\left(g_{i}\right)=m_{i}\left(m_{i}-1\right)$ and $\operatorname{dim}\left(M_{i}\right)=m_{i}$. In the first step, we set $M^{0+}:=M_{0}^{m_{0}}$ with metric $G^{0}:=g_{0}$. And then for $1 \leq s \leq \ell$ we define recursively

$$
G^{s}:=r_{s}^{-2}\left(d r_{s}^{2}+\left(1+r_{s}^{2} / 4\right)^{2} \cdot G^{s-1}+\left(1-r_{s}^{2} / 4\right)^{2} \cdot g_{s}\right),
$$


which is a metric on the interior of $\underline{M}^{s}:=M^{(s-1)+} \times M_{s} \times I_{s}$, where $I_{s}=[0,2)$ is an interval of length $\sqrt{2 /|\mu|}=2$ with parameter $r_{s}$. The interior of $\underline{M}^{s}$ is given by $M^{s+}:=M^{(s-1)+} \times M_{s} \times(0,2)$. Using Theorem 4.1 inductively for every step of the construction proves the following result on multiple sub-products.

Theorem 8.1. Let $\left(\underline{M}^{\ell}, G^{\ell}\right), \ell \geq 1$, be recursively defined as above. Then the metric $G^{\ell}$ on $M^{\ell+}$ is Poincaré-Einstein with dimension $\operatorname{dim}\left(\underline{M}^{\ell}\right)=$ $-\left(s+\sum_{i=0}^{s} m_{i}\right)$. The conformal infinity is described by

$$
\left(M^{(\ell-1)+} \times M_{\ell},\left[G^{\ell-1} \times g_{\ell}\right]\right) .
$$

An ambient metric of the conformal structure $\left[G^{\ell-1} \times g_{\ell}\right]$ is explicitly given by

$$
h^{\ell}:=\bar{g}_{0} \times \cdots \times \bar{g}_{\ell}
$$

on $M_{0} \times \cdots \times M_{\ell} \times \mathbb{R}_{+}^{\ell+1}$, where the $\bar{g}_{i}$ 's denote the cone metrics of the $g_{i}$ 's. By Theorem 3.4 we can conclude that the conformal structure $\left[G^{\ell-1} \times g_{\ell}\right]$ at infinity is not conformally almost-Einstein in case that every metric $g_{i}$ for $i \in\{0, \ldots, \ell\}$ of the initial setting admits exactly one almost-Einstein scale.

\section{REFERENCES}

[1] Pierre Albin, Renormalizing Curvature Integrals on Poincare-Einstein Manifolds, math.DG/0504161.

[2] M. Anderson, $L^{2}$ curvature and volume renormalization of AHE metrics on 4manifolds. Math. Res. Lett. 8 (2001), no. 1-2, 171-188.

[3] Stuart Armstrong, Definite signature conformal holonomy: a complete classification, math.DG/0503388.

[4] Stuart Armstrong, and Thomas Leistner, Ambient connections realising conformal Tractor holonomy, math.DG/0606410

[5] R. Bach, Zur Weylschen Relativitätstheorie und der Weylschen Erweiterung des Krümmungstensorbegriffs, Math. Z. 9 (1921), 110-135.

[6] T.N. Bailey, M.G. Eastwood, and A.R. Gover, Thomas's structure bundle for conformal, projective and related structures, Rocky Mountain J. Math. 24 (1994), 11911217.

[7] O. Biquard, Métriques d'Einstein asymtotiquement symmétriques, Astérisque 265 (2000).

[8] A. Čap, and A.R. Gover, Tractor calculi for parabolic geometries, Trans. Amer. Math. Soc., 354 (2002), 1511-1548.

[9] Andreas Čap, A. Rod Gover, Standard tractors and the conformal ambient metric construction, Ann. Global Anal. Geom. 24 (2003), 231-259.

[10] E. Cartan, Les espaces à connexion conforme, Ann. Soc. Pol. Math. 2 (1923), 171202.

[11] Alice Chang, Jie Qing, Paul Yang On the renormalized volumes for conformally compact Einstein manifolds, math.DG/0512376

[12] C. Fefferman and C.R. Graham, Conformal invariants. In "Élie Cartan et les Mathématiques d'Adjourd'hui" (Astérisque, hors serie), 1985, pp. 95-116.

[13] Charles Fefferman, C. Robin Graham, Q-curvature and Poincaré metrics, Math. Res. Lett. 9 (2002), 139-151.

[14] Charles Fefferman, and Kengo Hirachi, Ambient metric construction of Q-curvature in conformal and CR geometries, Math. Res. Lett. 10 (2003), 819-831.

[15] G.W. Gibbons, and P. Rychenkova, Cones, tri-Sasakian structures and superconformal invariance, Phys. Lett. B 443 (1998), 138-142.

[16] A.R. Gover, Almost conformally Einstein manifolds and obstructions, in Differential Geometry and its Applications, Proceedings of the 9th International Conference on 
Differential Geometry and its Applications, Prague 2004, Charles University, Prague, 2005, pp243-255. Electronic: math.DG/0412393, http://www.arxiv.org

[17] A.R. Gover Laplacian operators and Q-curvature on conformally Einstein manifolds, 336, (2006) 311-334. Preprint math.DG/0506037, http://arXiv.org.

[18] A.R. Gover and L.J. Peterson, Conformally invariant powers of the Laplacian, Qcurvature, and tractor calculus,, Commun. Math. Phys. 235 (2003) 339-378.

[19] A.R. Gover and L.J. Peterson, The ambient obstruction tensor and the conformal deformation complex, Pacific J. Math. 226 (2006), no. 2, 309-351. math.DG/0408229, http://arXiv.org

[20] C. Robin Graham, Volume and area renormalizations for conformally compact Einstein metrics, Rend. Circ. Mat. Palermo (2) Suppl. No. 63 (2000), 31-42.

[21] C.R. Graham, R. Jenne, L.J. Mason, G.A. Sparling, Conformally invariant powers of the Laplacian. I. Existence, J. London Math. Soc. (2) 46 (1992), 557-565.

[22] C.R. Graham and K. Hirachi, The ambient obstruction tensor and $Q$-curvature, in AdS/CFT correspondence: Einstein metrics and their conformal boundaries, 59-71, IRMA Lect. Math. Theor. Phys., 8, Eur. Math. Soc., Zrich, 2005.

[23] C. Robin Graham, and John M. Lee, Einstein metrics with prescribed conformal infinity on the ball Adv. Math. 87 (1991), 186-225.

[24] C. Robin Graham, and Edward Witten, Conformal anomaly of submanifold observables in AdS/CFT correspondence, Nuclear Phys. B 546 (1999), 52-64.

[25] C. Robin Graham, and Maciej Zworski, Scattering matrix in conformal geometry, Invent. Math. 152 (2003), 89-118.

[26] C.R. LeBrun, $\mathcal{H}$-space with a cosmological constant, Proc. Roy. Soc. London Ser. A 380 (1982), 171-185.

[27] John M. Lee, Fredholm Operators and Einstein Metrics on Conformally Compact Manifolds, math.DG/0105046.

[28] Thomas Leistner, Conformal holonomy of C-spaces, Ricci-flat, and Lorentzian manifolds, math.DG/0501239.

[29] Felipe Leitner, Conformal holonomy of bi-invariant metrics, math.DG/0406299.

[30] Felipe Leitner, Conformal Killing forms with normalisation condition, Rend. Circ. Mat. Palermo (2) Suppl. No. 75 (2005), 279-292. e-print: math.DG/0406316.

[31] Felipe Leitner, A remark on conformal $S U(p, q)$-holonomy, math.DG/0604393

[32] J. Maldacena, The large $N$ limit of superconformal field theories and supergravity, Adv. Theor. Math. Phys. 2 (1998), 231-252.

[33] Rafe Mazzeo, and Frank Pacard, Maskit combinations of Poincare-Einstein metrics, math.DG/0211099.

[34] Barrett O'Neill, Semi-Riemannian geometry. With applications to relativity. Pure and Applied Mathematics, 103. Academic Press, Inc., New York, 1983.

[35] Uwe Semmelmann, Conformal Killing forms on Riemannian manifolds, Math. Z. 245 (2003), no. 3, 503-527.

[36] Georges deRham, Sur la reductibilité d'un espace de Riemann, Comment. Math. Helv. 26, (1952), 328-344.

Department of Mathematics, The University of Auckland, Private Bag 92019, Auckland 1, New Zealand

E-mail address: gover@math.auckland.ac.nz

Universitat Stuttgart, Institut fr Geometrie und Topologie, Mathematics

Department, Pfaffenwaldring 57, D-70550 Stuttgart

E-mail address: leitner@mathematik. uni-stuttgart.de 